\title{
Hadronic final states in deep-inelastic scattering with Sherpa
}

\author{
Carli, T ; Gehrmann, T ; Höche, S
}

\begin{abstract}
We extend the multi-purpose Monte Carlo event generator Sherpa to include processes in deeply inelastic lepton-nucleon scattering. Hadronic final states in this kinematical setting are characterised by the presence of multiple kinematical scales, which were up to now accounted for only by specific resummations in individual kinematical regions. Using an extension of the recently introduced method for merging truncated parton showers with higher-order tree-level matrix elements, it is possible to obtain predictions which are reliable in all kinematical limits. Different hadronic final states, defined by jets or individual hadrons, in deep-inelastic scattering are analysed and the corresponding results are compared to HERA data. The various sources of theoretical uncertainties of the approach are discussed and quantified. The extension to deeply inelastic processes provides the opportunity to validate the merging of matrix elements and parton showers in multi-scale kinematics inaccessible in other collider environments. It also allows to use HERA data on hadronic final states in the tuning of hadronisation models.
\end{abstract}

DOI: https://doi.org/10.1140/epjc/s10052-010-1261-2

Posted at the Zurich Open Repository and Archive, University of Zurich

ZORA URL: https://doi.org/10.5167/uzh-34353

Journal Article

Accepted Version

Originally published at:

Carli, T; Gehrmann, T; Höche, S (2010). Hadronic final states in deep-inelastic scattering with Sherpa. European Physical Journal C - Particles and Fields, 67(1-2):73-97.

DOI: https://doi.org/10.1140/epjc/s10052-010-1261-2 
ZU-TH 20/09

MCNET/09/19

\title{
Hadronic final states in deep-inelastic scattering with SHERPA
}

\author{
Tancredi Carli ${ }^{1}$, Thomas Gehrmann², Stefan Höche ${ }^{2}$ \\ ${ }^{1}$ CERN, Department of Physics, CH-1211 Geneva 23, Switzerland \\ ${ }^{2}$ Institut für Theoretische Physik, Universität Zürich, CH-8057 Zürich, Switzerland
}

\begin{abstract}
We extend the multi-purpose Monte-Carlo event generator SHERPA to include processes in deeply inelastic lepton-nucleon scattering. Hadronic final states in this kinematical setting are characterised by the presence of multiple kinematical scales, which were up to now accounted for only by specific resummations in individual kinematical regions. Using an extension of the recently introduced method for merging truncated parton showers with higher-order tree-level matrix elements, it is possible to obtain predictions which are reliable in all kinematical limits. Different hadronic final states, defined by jets or individual hadrons, in deep-inelastic scattering are analysed and the corresponding results are compared to HERA data. The various sources of theoretical uncertainties of the approach are discussed and quantified. The extension to deeply inelastic processes provides the opportunity to validate the merging of matrix elements and parton showers in multi-scale kinematics inaccessible in other collider environments. It also allows to use HERA data on hadronic final states in the tuning of hadronisation models.
\end{abstract}

\section{Introduction}

Deep-inelastic lepton-nucleon scattering (DIS) allows to analyse the structure of the nucleon by means of a pointlike probe, and provides an experimental framework for a multitude of studies of strong interaction dynamics. The kinematical situation of deeply inelastic events offers access to configurations which cannot be probed at other colliders. The (space-like) virtuality of the exchanged photon sets a hard scale for the scattering process, which is also the unique hard scale in inclusive deep-inelastic structure functions. Studying more exclusive properties of the hadronic final state allows access to multiple other scales, given for example by the transverse momenta of final-state jets. Such multiple-scale configurations are impossible to realise in $e^{+} e^{-}$annihilation, where the centre-of-mass energy is the only hard scale of the process, and difficult to access in purely hadronic collisions (vector-boson plus multi-jet production being an example of such a multi-scale configuration). Hadronic final states in DIS thus offer the unique opportunity to study multi-scale processes in QCD and provide the advantage of a relatively clean experimental setting. A wealth of corresponding experimental data is available from the HERA collider experiments H1 and ZEUS.

The kinematical situation of a deeply inelastic scattering process with incoming proton momentum $p$ and incoming and outgoing electron momenta $k$ and $k^{\prime}$ is characterised by the virtuality $Q^{2}$ of the exchanged boson, carrying momentum $q=k-k^{\prime}$, and by the Bjørken variable $x$, which can be inferred purely from 
the outgoing electron momentum as

$$
Q^{2}=-q^{2}=\left(k-k^{\prime}\right)^{2} \quad \text { and } \quad x=\frac{Q^{2}}{2 q \cdot p}
$$

Measurements are usually either performed in the Breit frame, or in the centre-of-mass frame of proton and virtual photon, called the hadronic centre-of-mass frame. ${ }^{1}$ The centre-of-mass energy squared is then given by $W^{2}=Q^{2}(1-x) / x$. In lepton-hadron collisions, one generally distinguishes photoproduction processes, where the exchange photon is quasi-real, $Q^{2} \rightarrow 0$ with $W^{2}$ fixed, and deeply inelastic processes. This distinction is made experimentally by imposing a minimum cut on $Q^{2}$, typically of the order a few $\mathrm{GeV}^{2}$.

Inclusive structure functions as the basic quantities in deep-inelastic processes depend on $x$ and $Q^{2}$ only, and the description of the proton structure in terms of parton distributions is formulated in the space of these variables. The evolution of the parton distributions with increasing $Q^{2}$ is determined by the DokshitzerGribov-Lipatov-Altarelli-Parisi (DGLAP) equations [1], which are known to next-to-next-to-leading order [2] in QCD. These equations form the basis of the QCD-improved parton model, and allow for a determination of the process-independent parton distribution functions from global fits $[3,4,5,6]$ to data from lepton-hadron and hadron-hadron collisions. Higher-order corrections to the DGLAP equations [2] contain powers of logarithms in $x$ or $(1-x)$, which potentially spoil the convergence of the perturbative expansion at large and small $x$. In both limits, resummation formalisms for the large logarithmic corrections are available: threshold resummation at large $x$ and BFKL resummation [7] at small $x$. A unified DGLAP/BFKL resummation is provided by the CCFM equation [8]. The currently available inclusive structure function data can however be described entirely by the DGLAP framework. Considerable experimental and theoretical effort was made especially in order to establish observables sensitive to BFKL effects. In this context, specific hadronic final states, such as forward jets [9] are usually investigated and jet rapidity correlations appear to be promising observables.

Among hadronic final states, jet cross sections offer the most direct probes of parton-level dynamics. The H1 and ZEUS experiments have performed many different measurements of jet production processes, ranging from single-jet-inclusive and di-jet (which is often called $(2+1)$-jet because of the extra proton remnant jet) cross sections to multi-jet cross sections and jet correlations. While the former are used for precision determinations of the strong coupling and of parton distributions, the latter offer detailed insight into the production dynamics, and can highlight the kinematical limitations of the DGLAP framework. In this framework, next-to-leading order (NLO) QCD predictions are available for single-jet-inclusive and di-jet cross sections [10] and for three-jet production [11]. For central jet production, and provided that $Q^{2}$ is not much smaller than the transverse energies of the jets in the Breit frame, $E_{T, B}$, these calculations yield a very good description of the experimental data [12]. In the situation of $Q^{2} \ll E_{T, B}^{2}$, large logarithmic corrections of the form $\ln \left(Q^{2} / E_{T, B}^{2}\right)$ appear to all orders in perturbation theory. By attributing a parton content to the virtual photon entering the hard process [13], these can be resummed. Including the contributions from the virtual photon structure into the NLO QCD calculations [14] extends the kinematical range where those are applicable (including the description of forward jet production [15]), and allows for a smooth transition from deep-inelastic to photoproduction processes.

Within the framework of Monte-Carlo event generation, multi-jet production is usually described through parton showers, starting from a leading-order process. Various parton-shower models exist, which are either based on DGLAP evolution [16,17,18,19], or CCFM evolution [20]. Other methods use the colour dipole model [21] or an approach based on the Catani-Seymour subtraction technique [22,23]. All of those models have in common, that they are capable of describing parton-level final states in certain regions of the phase space only, which are defined by the respective resummation prescription. By construction, none of them allows to correctly account for multi-parton correlations, and therefore their predictions should be corrected using higher-order matrix elements. Studies of electroweak gauge boson production [24] and the production of coloured heavy states [25] indicate that an improved description of high transverse momentum jets in the DGLAP framework can be obtained by an appropriate combination of tree-level matrix elements and parton showers [26,27]. Such merged calculations are reliable in most kinematical limits. They can be thought of as unifying the leading logarithmic expressions of the different resummation prescriptions (DGLAP, BFKL, virtual photon structure) in a single calculation. Recently, new and powerful techniques have been developed, which extend those methods [28,29]. Their advantage is that parton shower radiation patterns are recovered

1 The hadronic centre-of-mass frame is defined by $\vec{q}+\vec{p}=0$, while in the Breit frame $2 x \vec{p}+\vec{q}=0$. The two frames are related by a longitudinal boost. 


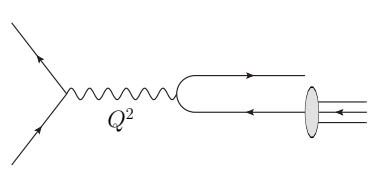

(a)

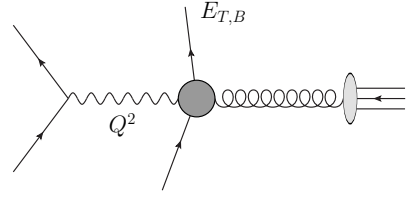

(b)

Figure 1: Schematic view of the scattering kinematics in the Breit frame for leadingorder $e^{ \pm} q \rightarrow e^{ \pm} q$ scattering and 2-jet production processes in DIS. The lightly shaded blob denotes the incoming proton. For 2-jet events with large jet transverse energy, $E_{T, B}^{2} \gtrsim Q^{2}$, the $2 \rightarrow 2$ process depicted by the dark shaded blob in Fig. (b) sets the hard scale.

to the accuracy provided by the shower model and therefore any statements about the logarithmic accuracy of the parton shower also holds for a merged calculation in this scheme. These novel techniques have so far been used in two relevant cases, namely $e^{+} e^{-}$annihilation into hadrons and Drell-Yan like production of electroweak gauge bosons. The technical prerequisites for realising the approach in [28] were implemented into the multi-purpose Monte-Carlo event generator SHERPA [30] in full generality. Since hadronic final states in deep-inelastic scattering depend on multiple kinematical scales, related observables provide an independent and particularly sensitive test of the quality of the approach and its implementation. It is the aim of this work to present a first study of this class of processes with SHERPA, and to confront results with data from the HERA experiments. Moreover we propose an extension of the merging algorithm in [28], which accounts for the proper simulation of low- $Q^{2}$, high- $E_{T, B}^{2}$ events.

Hadronic final states also offer insight into strong interaction dynamics at lower scales. Using kinematical spectra of identified hadrons, it is possible to probe the parton-to-hadron transition (hadronisation), which can not be computed from first principles, but is usually described using semi-empirical models [31, 32, 33]. These models are typically tuned to data from $e^{+} e^{-}$collider experiments and the obtained fits are assumed to be universal. Including data from deep-inelastic processes instead allows to probe different flavour combinations and beam remnant fragmentation, and to resolve parameter degeneracies. It is therefore vital to have means for simulating partonic final states in deep-inelastic processes reliably, not only to describe jet spectra, but also to reduce uncertainties in fragmentation models, which can then be tuned to experimental data in a combined fit. Therefore we also provide some examples for the influence of the parton-level inputs on different fragmentation models.

The outline of this paper is as follows. Section 2 introduces the technical details of the Monte Carlo simulation, including the proposal of an improved merging technique for low- $Q^{2}$ events. Section 3 presents the results of our analysis and discusses theoretical uncertainties. Finally Sec. 4 contains some concluding remarks.

\section{Event generation techniques}

The most striking difference between deep-inelastic scattering and processes like Drell-Yan lepton pair production is the nearly arbitrary hard scale $Q^{2}$, at which the proton structure can be probed by the virtual photon. While this presents an excellent opportunity for measuring the QCD dynamics of the process, it also constitutes the main obstacle for simulating it with Monte Carlo techniques. The reason for these problems and the solution adopted in the context of this work are outlined below.

\subsection{Parton shower evolution}

Existing parton shower simulations are often based on virtuality ordering $[16,19]$ or transverse momentum ordering $[18,23,22]$. The hard scale, i.e. the maximum evolution parameter for a set of colour connected partons, is then usually taken to be the maximum virtuality involved in the production of these partons. In Drell-Yan like events, for example, the hard scale is taken as the invariant mass of the final state lepton pair. In the case of DIS it is taken as the (negative) photon virtuality $Q^{2}$. Using this choice it is a priori impossible to fill the complete available phase space with parton-shower emissions, since $Q^{2}$ tends to be close 
to zero. Next-to-leading order calculations indicate, however, that the emission probability for additional partons is large, even if $Q^{2}$ is low, due to the possibly large hadronic centre-of-mass energy.

The leading contribution in this context stems from the interaction, $e^{ \pm} g \rightarrow e^{ \pm} q \bar{q}$, where the "sub-process" $\gamma^{*} g \rightarrow q \bar{q}$ plays the role of the hardest interaction, if the transverse energy squared of the final state quarks in the Breit frame, $E_{T, B}^{2}$, is larger than $Q^{2}$ (cf. Fig. 0). A similar problem was noted in [34] in the context of supersymmetric particle production at hadron colliders. To circumvent it, and allow a wider range of the phase space to be accessible for parton shower radiation, so-called "power shower" schemes were employed to artificially increase the starting scale of the initial-state shower. Although apparently in conflict with factorisation assumptions, this approach has recently received some theoretical support from the proposition of modified DGLAP evolution equations [35].

Another approach to overcome the restriction of the shower phase space by a low factorisation scale would be to employ an ordering parameter different from virtuality or transverse momentum; one which is more suited for the description of radiation off colour dipoles connected to initial-state hadrons. In fact, the proper framework for treating initial-state radiation is given by the CCFM equations [8], which order branchings in terms of emission angles, cf. also [17]. The CCFM scheme allows the transverse momentum of emitted partons to become larger than the factorisation scale and can therefore provide a generic solution of the above problem. It has successfully been used in several Monte-Carlo event generators [20]. We do, however, not resort to the CCFM technique here. The fact that a transverse momentum ordered parton shower can only sensibly describe parton spectra below the factorisation scale will rather be compensated by a special technique for merging matrix elements and truncated showers, which is introduced in Sec. 2.2.

In the context of this work we employ the parton-shower algorithm initially presented in [22], which is based on the Catani-Seymour (CS) subtraction method, cf. [36]. Modifications of the original approach to account for recoil effects into the final state from splitting initial state partons with final state spectator were recently proposed [37] and are refined in [38]. It is interesting to investigate the corresponding effect on the parton shower predictions. Figure 1a shows differential $n$-jet rates, i.e. the scale where an $n$-jet event is clustered into an $n-1$-jet event, using the exclusive $k_{T}$-jet algorithm [39]. The difference between the predictions are sizeable, when switching between the original and the modified recoil scheme, especially in the low- $k_{T}$ domain, $1 \mathrm{GeV} \lesssim k_{T} \lesssim 10 \mathrm{GeV}$. This implies that the choice of the recoil scheme in a given partonshower simulation should be part of an uncertainty analysis, much like the variation of renormalisation and factorisation scales. We comment on this subject in Secs. 2.2 and 3. To improve the parton-shower prediction in the domain of hard emissions and therefore alleviate the merging with NLO real emission matrix elements, the shower splitting kernels can be modified to include matrix element corrections. The corrected splitting kernels amount to antenna functions [40], which were used for parton showers only in $e^{+} e^{-}$annihilation up to now [41]. The corresponding procedure is outlined in Appendix A. Figures 1b and 1c show the influence of these corrections on the $k_{T}$-jet rates in the Breit frame. We observe a substantial change in the total rate of emissions. In the following, matrix element corrected splitting kernels are therefore employed.

\subsection{Merging matrix elements and parton showers}

Next-to-leading order calculations and parton-shower simulation present two essentially different approaches to perturbative QCD. Fixed-order calculations seek to determine all finite corrections to the leading-order process and are usually most important when measuring inclusive quantities. Parton-shower simulation aims at a proper resummation of large logarithmic corrections to the leading-order result, while preserving the overall cross section of the initial event sample. For the first emission this presents an approximation to the real NLO correction, whose quality largely depends on the underlying assumptions about the splitting kinematics and the recoil scheme, as outlined in Sec. 2.1. Thus, parton-shower simulations are inherently incapable to describe the precise correlations between more than a few final-state QCD particles properly. While the number of particles of interest is still low $(\mathcal{O}(1-6))$, the corresponding problems can easily be corrected by employing full tree-level matrix elements instead of splitting kernels. Their computation has been automated in various approaches and poses no conceptional problem. The task is then reduced to finding an efficient and versatile algorithm for implementing the parton-shower correction in a generic way. Several methods attempted to solve this problem in the past $[26,42,27]$, while two especially suitable approaches were suggested only recently $[28,29]$. In the context of this work, the shower-independent formulation in terms of truncated parton showers and an arbitrary jet criterion is employed, which was introduced in [28]. The basic idea is to separate the phase space into a matrix-element and a parton-shower domain through 


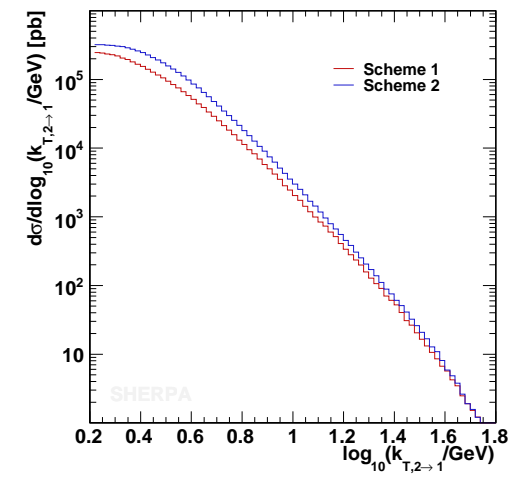

(a)

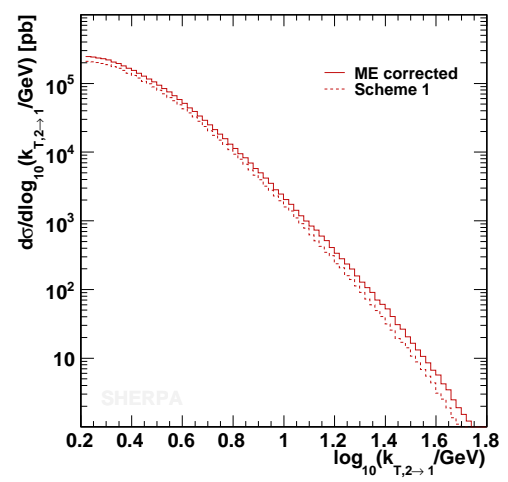

(b)

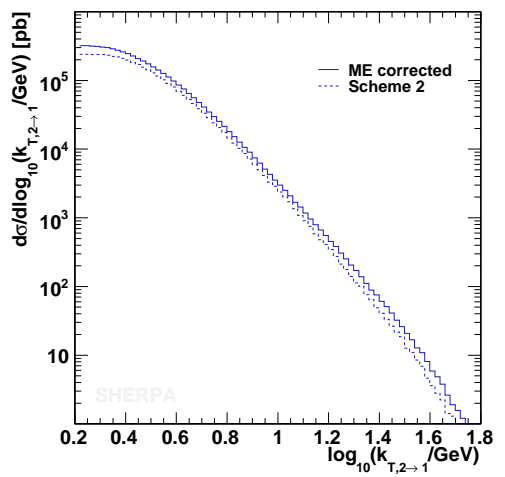

(c)

Figure 2: Differential 2-jet rates defined by the exclusive $k_{T^{-}}$jet algorithm in the Breit frame for deepinelastic scattering events with $Q^{2}>4 \mathrm{GeV}^{2}$. Part (a) compares the influence of different recoil strategies, while parts (b) and (c) show the effect of matrix element corrections. Monte Carlo samples were generated using the parton shower model of [22]. Scheme 1 stands for the recoil strategy in $[37,38]$, while scheme 2 labels the original strategy employed in [22].

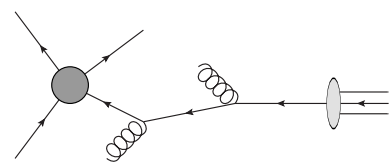

(a)

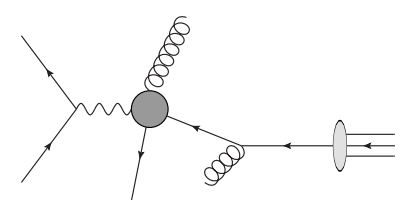

(b)

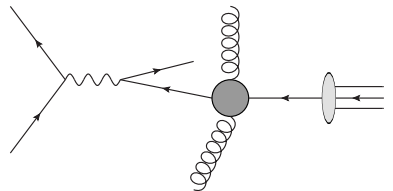

(c)

Figure 3: Schematic view of three possible core process choices in DIS three-jet production. Part (a) corresponds to the most probable core process being the virtual photon exchange, while additional hard partons are interpreted as parton shower emissions. Parts (b) and (c) depict configurations, where the most probable core process is the interaction of the virtual photon with a parton and a pure QCD $2 \rightarrow 2$ process, respectively.

a cut in the real-emission phase space. The matrix-element domain is then supposed to contain hard, well-separated partons only, while the parton-shower domain covers the region where resummation effects become important. Throughout the hard domain parton-shower emissions are corrected using tree-level matrix elements up to a given maximum multiplicity. In the soft domain, the parton shower is applied as is. The separation is achieved in terms of a so-called jet criterion, defining the "hardness" and/or the separation of a parton with respect to others [28]. This can be thought of as a kind of $k_{T}$-jet measure, cf. e.g. [39].

As pointed out in [28], this merging algorithm needs to be refined if the scale difference between $Q^{2}$ and the hardness scale $k_{T}^{2}$ of additional partons is large and negative. In this case, logarithmic corrections are not induced by $Q^{2} / q^{2}$, but rather by $k_{T}^{2} / q^{2}$, where $q^{2}$ is the jet resolution scale. In the case of DIS, the production of the virtual photon can then be viewed as an electroweak splitting process, attached to the core $\gamma^{*} j \rightarrow j j$ interaction, as depicted in Fig. 2b. In the extreme case of very hard jets, the core process does not even include the virtual photon. This is visualised in Fig. 2c. The correct choice of the core process is not arbitrary, but is rather fixed by the backwards clustering algorithm described in [28], cf. also [43]. To allow an inclusive merging procedure, the clustering algorithm must allow to identify the virtual photon as a soft particle, which is removed in order to find the core process and reproduced later in unfolding the matrix-element branching history. If QED splitting functions are included into the parton-shower, the correct method is obtained immediately, cf. also [38].

The above merging algorithm can also be employed to solve the problem outlined in Sec. 2.1. That is, it can be used to fill the complete available real emission phase space for any given $Q^{2}$. A similar solution is in fact adopted in Drell-Yan lepton-pair production via $\gamma^{*} / Z$-exchange, where the separation cut $Q_{\text {cut }}$ between 
matrix-element and parton-shower domain is set such that $Q_{\text {cut }}<m_{l l^{\prime}}$, with $m_{l l^{\prime}}$ being the invariant mass of the lepton pair. This situation is particularly simple, since an experimental cut is usually applied, which enforces $m_{l l^{\prime}} \approx m_{Z}$. Therefore $Q_{\text {cut }}$ can remain constant at $Q_{\text {cut }}=S_{\mathrm{DY}} m_{Z}$, where $S_{\mathrm{DY}}$ is an in principle arbitrary constant with $0<S_{\mathrm{DY}}<1$. Of course, $S_{\mathrm{DY}}$ must be chosen sensibly, such as not to drive $Q_{\text {cut }}$ into the non-perturbative domain. Also, $S_{\mathrm{DY}}$ should not be too close to one, since the proper description of particle spectra in this region largely depends on the recoil strategy employed in the shower. In practice, we have $0.1 \lesssim S_{\mathrm{DY}} \lesssim 0.5$. In deep-inelastic-scattering the situation is slightly different due to the variable value of $Q^{2}$. The solution can, however, be identical. We choose

$$
Q_{\text {cut }}=\bar{Q}_{\text {cut }}\left[1+\frac{\bar{Q}_{\text {cut }}^{2} / S_{\text {DIS }}^{2}}{Q^{2}}\right]^{-1 / 2},
$$

where $\bar{Q}_{\text {cut }}$ is a fixed value, much like $Q_{\text {cut }}$ in the Drell-Yan pair production case. It ensures that high- $Q^{2}$, medium- $E_{T, B}^{2}$ events are described by matrix elements, rather than by the parton shower. At the same time, the factor in the square bracket, including $S_{\text {DIS }}<1$, enforces low- $Q^{2}$, high- $E_{T, B}^{2}$ events to be in the matrix-element domain as well, such that the complete available real-emission phase space can be filled by the Monte-Carlo simulation. Note that, contrary to the large freedom in the choice of $\bar{Q}_{\text {cut }}$, we are rather limited in the choice of $S_{D I S}$. Most analyses of deep-inelastic scattering data employ a cut on the photon virtuality which is of the order of a few $\mathrm{GeV}^{2}$. The Monte-Carlo simulation, however, is bound to have $Q_{\text {cut }}$ in the perturbative domain with some difference between $Q_{\text {cut }}^{2}$ and $Q^{2}$, as discussed above. This introduces rather strict limits on the available range for $S_{\text {DIS }}$. To be specific,

$$
0.4 \lesssim S_{\text {DIS }} \lesssim 0.8
$$

where the lower bound depends on the experimental setup and the upper bound depends on the partonshower model.

Figure 3 illustrates the effect of different $\bar{Q}_{\text {cut }}$ and different $S_{\text {DIS }}$ on the prediction for the differential 2- and 3jet rates in the Breit frame. The Monte-Carlo result remains very stable against corresponding variations. It can also be seen that when merging the parton shower with matrix elements, previous differences arising from different recoil strategies reduce considerably. This is essentially because the parton-shower contribution to the observable is largely reduced, such that kinematical effects from shower branchings have far less influence than in event samples without matrix-element merging.

\section{Comparison with experimental data}

In this section, Monte-Carlo predictions, generated according to Secs. 2.1 and 2.2, are confronted with hadronic final state data taken by the $\mathrm{H} 1$ experiment. The correct description of the selected measurements is quite challenging for the Monte Carlo traditionally used in the analysis of HERA data [44]. We seek to quantify the effect of varying perturbative input parameters and varying intrinsic parameters of the merging approach. We are mainly interested in the hard, perturbative domain, and therefore we choose to focus particularly on jet analyses. Monte-Carlo predictions stem from the SHERPA program [30], which, in this context, employs the matrix-element generator COMIX [45] to simulate the hard processes. Parton showers are implemented by the dipole-like cascade presented in [22]. Hadronisation is simulated either using the cluster fragmentation model of SHERPA [33], or the Lund string fragmentation model [31] in the implementation of PYTHIA [46]. Both models were previously tuned to describe LEP data employing the PROFESSOR program [47]. ${ }^{2}$ Hadron decays are implemented by SHERPA's internal hadron decay module [48] or by PYTHIA [46], depending on the hadronisation model employed. Photon radiation is simulated by SHERPA's internal YFS generator [49]. All analyses are carried out using the HZTool library [50].

If not stated otherwise, matrix elements with up to five QCD partons in the final state are employed and the parameters of the matrix-element parton-shower merging according to Sec. 2.2 are set to $S_{\text {DIS }}=0.6$ and $\bar{Q}_{\text {cut }}=5 \mathrm{GeV}$. The default PDF set is NNPDF $1.2[4]$ in the implementation with 100 replicas. The perturbative order of the strong coupling and its value at the reference scale $m_{Z}$ is always chosen in accordance with the PDF set.

\footnotetext{
${ }^{2}$ We are indebted to Frank Krauss, Hendrik Hoeth and Eike von Seggern for making preliminary sets of tuning parameters available.
} 

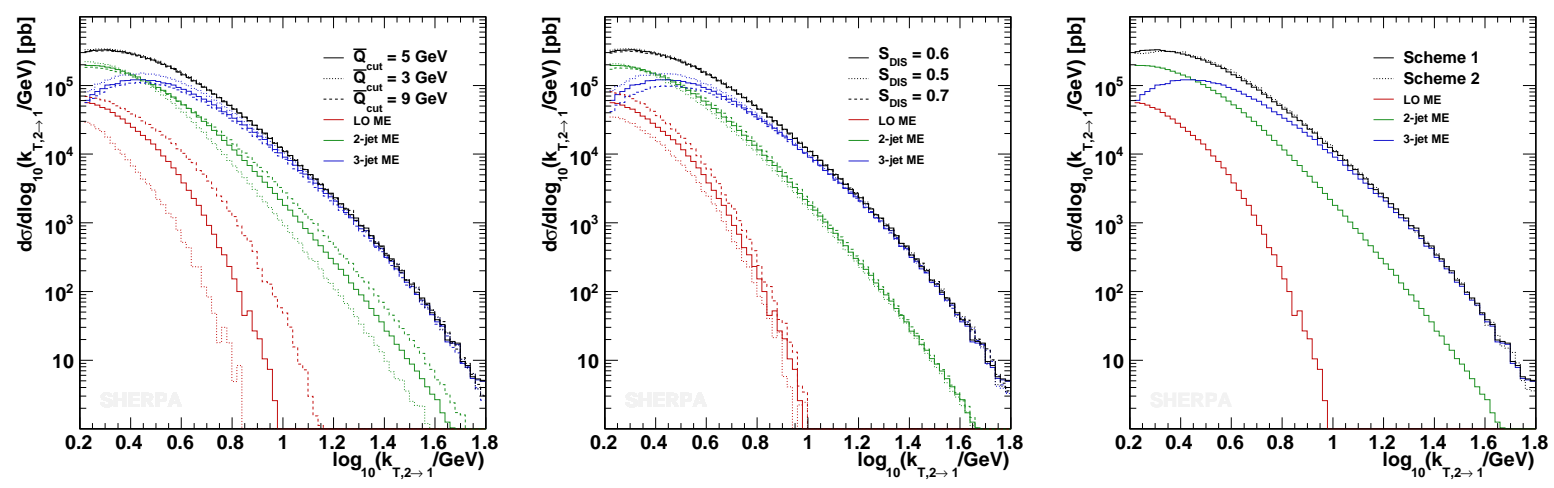

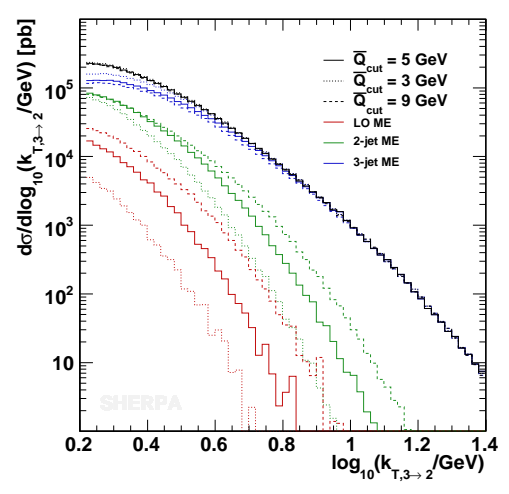

(a)

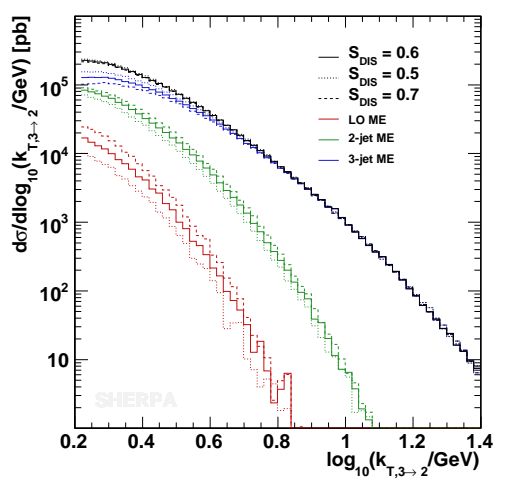

(b)

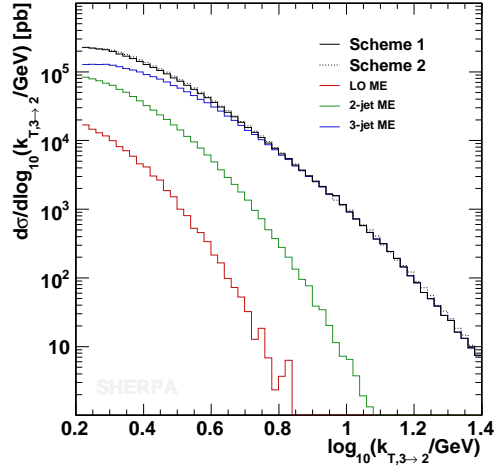

(c)

Figure 4: The differential 2- and 3-jet rates in merged event samples of varying $\bar{Q}_{\text {cut }}$ (a), varying $S_{\text {DIS }}$ (b) and varying shower recoil strategy (c). See also Fig. 1 for notation. Coloured lines display the contributions of different final state multiplicity matrix elements. The central parameter value is chosen as $\bar{Q}_{\text {cut }}=5 \mathrm{GeV}$ and $S_{\text {DIS }}=0.6$. The maximum parton multiplicity in hard matrix elements is $N_{\max }=3$.

\subsection{Inclusive jet analysis}

Following the arguments of Sec. 2.1 and 2.2, a crucial observable is given by the inclusive jet cross section, differential with respect to $E_{T, B}^{2} / Q^{2}$, where $E_{T, B}$ is the jet transverse energy in the Breit frame. For $E_{T, B}^{2} / Q^{2}>1$ it probes a part of the phase space where leading order Monte-Carlo models without the inclusion of low- $x$ effects are bound to fail in their description of jet spectra. The question to be answered here is, whether the incorporation of higher order tree-level matrix elements is sufficient to improve on this deficiency and yield predictions which are consistent with experimental data. Inclusive $E_{T, B}^{2} / Q^{2}$-spectra were measured for different ranges of the jet-pseudorapidity in the laboratory frame, $\eta_{l a b}$, in the low- $Q^{2}$ domain $5<Q^{2}<100 \mathrm{GeV}^{2}$ by the $\mathrm{H} 1$ collaboration [51]. In this analysis jets were defined using the inclusive $k_{T}$-algorithm [52] and were constrained to $E_{T, B}>5 \mathrm{GeV}$ and the pseudorapidity range $-1<\eta_{l a b}<2.8$. It was found that next-to-leading order QCD calculations can describe the data reasonably well, while large differences between leading-order and next-to-leading order results have been observed, especially in the forward region $1.5<\eta_{l a b}<2.8$.

Results from our analysis are compared to the $\mathrm{H} 1$ data in Figs. 5 to 7 . Figure 5 a shows that the Monte-Carlo prediction gradually improves with a growing number of final-state partons in the hard matrix elements. This behaviour is expected. Including matrix elements of larger final-state multiplicity corresponds to opening the full phase space for high- $E_{T, B}$ jet production. If all possible channels are to be incorporated, matrix elements with at least three final state partons must be available (3-parton sample). Indeed we observe that if only matrix elements with up to two final-state partons are considered (2-parton sample), the MonteCarlo prediction is far off the data. While the 3-parton sample gives an improved description, the data are described satisfactorily only by a 4 -parton sample. Including one additional emission in the matrix elements 
does not alter the results too much. This can be understood as an effect of the definition of the observable. Since inclusive jet spectra are investigated, the proper description of the kinematics of sub-leading jets can be as important as the simulation of the leading jet.

Theoretical uncertainties of the Monte-Carlo prediction are shown in Figs. 5b to 6b. The variation of renormalisation and factorisation scales has the largest impact on our results. This is expected, since an improved leading order approach is employed, which does not allow to predict the total cross section correctly. Higher-order virtual corrections are missing in this algorithm. However, the systematic inclusion of higherorder real corrections allows the prediction of the jet spectra in arbitrary multi-jet topologies at leading order, a feature which is inherently not present in any fixed-order calculation. The uncertainties associated with a variation of the intrinsic parameters of the merging algorithm are small compared to the variation found when altering the renormalisation and factorisation scale. This is exemplified in Fig. 5b.

Figure $7 \mathrm{a}$ shows that the uncertainty related to the parton shower recoil strategy is negligible. This is a direct consequence of the merging approach and has been discussed in Sec. 2.2, cf. Fig. 3. We also compare the influence of different PDF sets on the results of our analysis. All those PDFs are based on next-to-leading order fits. Figure $7 \mathrm{~b}$ shows that the corresponding results are essentially compatible, while a slight preference can currently be given to the NNPDF 1.2 set, cf. also Fig. $9 \mathrm{~b}$.

\subsection{Inclusive jet and di-jet analysis}

It is interesting to investigate jet properties in some more detail. The analysis presented in [53] covers a wider range of $Q^{2}$ and presents jet- $E_{T, B}$ spectra doubly differential in $Q^{2}$ and $E_{T, B}$. Jet pseudorapidities $\left(\eta_{l a b}\right)$ and pseudorapidity differences $\left(\eta^{\prime}=\left|\eta_{B, 1}-\eta_{B, 2}\right| / 2\right)$ were also analysed. Next-to-leading order calculations turn out to be particularly important for the latter, with deviations from leading-order predictions being most pronounced in the region of large $\eta_{l a b}$ of the forward jet. The acceptance region of this measurement is $5<Q^{2}<15000 \mathrm{GeV}^{2}$ and $-1<\eta_{l a b}<2.5$. Jet transverse energies are subject to the cuts $E_{T, B} 1,2>5 \mathrm{GeV}$ and $E_{T, B 1}+E_{T, B 2}>17 \mathrm{GeV}$. The latter requirement is introduced to avoid $E_{T, B 1} \approx E_{T, B 2}$, which is the region of the phase space where next-to-leading order corrections are unstable due to implicit restrictions on soft emissions [54].

A good probe of the proper Monte-Carlo simulation of such events is the di-jet cross section shown in Fig. 6 of [53]. While still a relatively inclusive quantity compared to the double differential spectra in the rest of this analysis, it tests the proper behaviour of jet production with decreasing $Q^{2}$ and is therefore as important as the jet- $E_{T, B}^{2} / Q^{2}$ spectra shown previously. It can be seen in Fig. 8a that a large number of partons simulated with hard matrix elements is needed to describe this observable properly. Once a good description is obtained, however, we are also capable of predicting the double differential jet spectra in $E_{T, B}$ and $\eta^{\prime}$, cf. Figs. 10 and 11. The agreement is excellent over the complete $Q^{2}$-range of the measurement, which implies that the merging approach is well capable to describe the dynamics of multi-jet final states, if the maximum multiplicity in hard matrix elements is large enough. We show theoretical uncertainties associated with our predictions of the $Q^{2}$-spectrum in Figs. $8 \mathrm{~b}$ to $8 \mathrm{~d}$. The same comments as for the previous section apply. It can also be seen in Fig. 8c, that a variation of renormalisation and factorisation scales does not only result in a global $K$-factor, i.e. a redefinition of the total cross section. Varying these scales can instead induce a distortion of jet- and particle spectra and it is therefore important to assess the related uncertainties.

\subsection{Low- $x$ di-jet analysis}

In DIS di-jet events, next-to-leading order corrections are especially large when the two jets in the Breit frame have similar transverse energy [54]. It is thus interesting to study an observable, which singles out the corresponding region of the phase space. A dedicated measurement which defines such an observable was carried out for the low- $Q^{2}$, low- $x$ domain by the H1 collaboration [55]. Jets were defined using the inclusive $k_{T}$-algorithm [52] and were constrained to $E_{T, B}>5 \mathrm{GeV}$ and the pseudorapidity range $-1<\eta_{l a b}<2.5$. Deep-inelastic scattering events were selected in the kinematic range $5 \mathrm{GeV}^{2}<\mathrm{Q}^{2}<100 \mathrm{GeV}^{2}$ and $10^{-4}<$ $x<10^{-2}$. A variable $\Delta$ was defined by the requirement $E_{T, \max }^{*}>E_{T \text { cut }}^{*}+\Delta$, where $E_{T \text { cut }}^{*}$ is the minimum jet transverse energy and $E_{T \text { max }}^{*}$ is the transverse energy of the hardest jet. All quantities marked with an asterisk are given in the hadronic centre-of-mass frame, which is related to the Breit frame simply by a longitudinal boost [55]. An observable $\Delta \eta^{*}$ was defined as the pseudorapidity difference between the two hardest jets in the event for a fixed value of $\Delta=2 \mathrm{GeV}$. 
The results of our Monte-Carlo analysis are compared to the H1 data in Figs. 12 and 13. For the $\Delta$ spectra we present parton level predictions and hadron level results. It can be seen that the effect of hadronisation on this observable is rather large in the region of very low $x$. The fact that hadronisation corrections are not uniform over the phase space indicates the importance of multi-purpose Monte-Carlo event generators. Full hadron-level events can easily be simulated by such programs. Also, the effect of different hadronisation models can be studied. We observe that the overall description of the data improves once hadronisation corrections are included. The fragmentation model employed here is the cluster fragmentation of [33].

\subsection{Three-jet analysis}

The three-jet analysis presented in [56] allows to test perturbative QCD predictions for events including one additional hard jet. Two angles, $\theta_{3}$ and $\psi_{3}$, were introduced in [57], which, together with the scaled energies of the jets, i.e. the jet energies w.r.t. the invariant mass of the three-jet system, can be used to parametrise the phase space of three-jet events. At the same time they exhibit some sensitivity to the correct simulation of the QCD dynamics, cf. $[57,56]$. They are defined in the three-jet centre-of-mass frame, with $\theta_{3}$ being the angle of the most energetic jet w.r.t. the proton beam direction and $\psi_{3}$ the angle between the plane containing the most energetic jet and the proton beam and the plane containing all three jets. The inclusive $k_{T}$-algorithm [52] was employed in [56] to define jets, which were then constrained to the region $E_{T, B}>5 \mathrm{GeV}$ and $-1<\eta_{l a b}<2.5$. Due to the construction of the $\mathrm{H} 1$ detector, the phase-space of the measurement is slightly different in the low- $Q^{2}\left(5 \mathrm{GeV}^{2}<\mathrm{Q}^{2}<100 \mathrm{GeV}^{2}\right)$ and in the high- $Q^{2}$ $\left(150 \mathrm{GeV}^{2}<\mathrm{Q}^{2}<5000 \mathrm{GeV}^{2}\right)$ analysis. Details can be found in the original publication.

Figure 14 compares the results of our Monte-Carlo analysis with data. The distribution of the angles $\theta_{3}$ and $\psi_{3}$ is relatively well described by the simulation. We also show the $Q^{2}$-distribution of the three-jet events, where the comments of Sec. 3.2 apply. We observe that the $Q^{2}$-spectrum is matched very well by the Monte-Carlo prediction, which again indicates the relevance of including high-multiplicity matrix elements into the simulation. A particularly useful observable to test the correct description of multi-jet rates, which is not available in di-jet events, is the ratio of the three- over the two-jet cross section, $R_{32}$. This quantity is independent of the overall normalisation of the event sample and is therefore especially suited to validate the Monte-Carlo models employed by leading-order event generators. We find satisfactory agreement with the corresponding data over the complete observed $Q^{2}$-range.

\subsection{Jet-shape analysis}

The analysis presented by the H1 collaboration in [58] investigates shapes and sub-jet rates of jets defined using either the inclusive $k_{T}$-algorithm [52] or a cone algorithm [59]. While the jet shape $\Psi(r)$ receives sizeable contributions from non-perturbative effects over the whole radial range of the jet, the sub-jet rate becomes fairly independent of non-perturbative dynamics at large values of the resolution parameter [58]. It is therefore a useful observable for measuring the perturbative dynamics of jet final states and can be used in particular to validate our Monte-Carlo simulation of parton evolution.

We exemplify in Figs. 15 and 16 that these observables are described satisfactorily by our Monte-Carlo approach. In fact, this can be expected once jet rates and event shapes are fitted in $e^{+} e^{-}$experiments. In this respect, we present a simple but necessary cross-check on the universality of the parton shower and hadronisation algorithms, which tests the nontrivial extension from pure final-state parton evolution to combined final- and initial-state evolution.

\subsection{Energy-flow analysis}

Energy flows are crucial observables to determine the properties of QCD final states in the region where perturbative and non-perturbative effects are equally important. It has been pointed out [60] that fragmentation might have as big an impact on these observables as has the perturbative input from hard processes and parton showering. This statement is supported by the observation that a large part of the spectra can be tuned to described the data by varying fragmentation parameters only. However, the influence of the perturbative input, i.e. the distribution of partons in the phase space and their colour correlations after the termination of the parton cascade, cannot be neglected. Transverse energy flows thus constitute an ideal observable to test the interplay between the hard, perturbative event phase and the hadronisation phase 
in Monte Carlo programs. The analysis presented by the H1 collaboration in [61] extended previous measurements to a larger $\eta$-range and higher $Q^{2}$, where the usage of a forward calorimeter (PLUG) allowed the determination of data points at very low $\eta^{*}$, the particle rapidity in the hadronic centre-of-mass frame. As pointed out in [61], the analysis of the transverse energy flow in this frame of reference isolates the physically interesting part of the distribution.

Figures 17 and 18 compare our Monte Carlo predictions to the H1 Data. We find very good agreement when employing the cluster fragmentation model of [33], while the Lund string fragmentation [31] gives predictions, which are slightly off the data. It should be noted, that a set of parameters can be found, with which the string fragmentation model gives better results for this particular observable. The fact that these parameters do not match those for which the model has been tuned to LEP data indicates the importance of a combined analysis when tuning intrinsic parameters of Monte-Carlo event generators.

\subsection{Charged particle spectra analysis}

Due to the large dependence of the transverse energy flows on hadronisation effects, transverse momentum spectra and pseudorapidity spectra of charged particles have been measured additionally by the H1 collaboration in [62]. It was argued in [63] that these observables provide a more direct measure of parton dynamics through a strong correlation between partons and final-state particles and might therefore be crucial to distinguish between DGLAP- and BFKL-driven parton evolution. The influence of hadronisation should be more pronounced in the low- $p_{T}$ region, while the high- $p_{T}$ tail of the distributions is more sensitive to perturbative effects. Significant discrepancies have been observed in the high- $p_{T}$ domain between the data and predictions from DGLAP-based Monte-Carlo models. Deviations also occur in the particle flow for $p_{T}>1$ $\mathrm{GeV}$ tracks.

We observe similar effects when comparing our Monte-Carlo results with the H1 data. Even though up to five-parton final states are included in our Monte-Carlo simulation, Fig. 19 shows discrepancies especially in the high- $p_{T}$ region. The particle flow for tracks with transverse momentum larger than $1 \mathrm{GeV}$, shown in Fig. 20, projects onto the critical part of the phase space. We show results from two different Monte-Carlo setups, labeled "Set 1" and "Set 2". While "Set 1" was produced using the cluster hadronisation model in combination with the NNPDF 1.2 PDF set "Set 2" displays predictions from the Lund string hadronisation in combination with the CTEQ 6L1 PDF set. We observed that the particle flow can be described satisfactorily by "Set 2". The corresponding results for other observables are, however, not matching the data. Using this parameterisation, for instance, transverse energy flows can not be described satisfactorily. Hence, at present, there is no agreement with data for these observables. This finding highlights the importance of including HERA data into the global tuning of hadronisation parameters.

\subsection{Charged multiplicity analysis}

Multiplicity distributions are one of the basic observables in hadronic final states. Much like the transverse energy flows, they allow a validation of the interplay between perturbative and nonperturbative parts of a Monte-Carlo simulation of detector events. The evolution of charged particle multiplicities with the hadronic centre-of-mass energy, $W$, and their dependence on the allowed pseudorapidity range was studied by the $\mathrm{H} 1$ collaboration in [64].

We present a comparison between our Monte-Carlo results and the data in Fig. 21. Good agreement over the complete $W$ and $\eta$ range is observed.

\section{Conclusions}

In this publication, we have extended the SHERPA event-generation framework to describe hadronic final states in deep-inelastic lepton-nucleon scattering processes. SHERPA is a modern event generator, which implements the merging of matrix-element based event generation with a parton shower.

The merging procedure relies on a backward clustering algorithm according to an inverted parton shower, which determines a hard core process at the origin of the matrix element or the parton shower. For a fully inclusive matrix-element parton-shower merging, the clustering must be performed on all outgoing particles. Depending on the final state kinematics, characterised by the photon virtuality $Q^{2}$ and the 
transverse momenta of final state jets, the core process in deep-inelastic scattering is then either found as electron-quark scattering, photon-quark scattering or a partonic $2 \rightarrow 2$ scattering process.

To account for the kinematical situation in deep-inelastic scattering, the merging procedure in SHERPA was refined, taking into account that most hadronic final states are characterised by several hard scales, not only by $Q^{2}$. By choosing appropriate merging scales, a successful description of processes in all kinematical regions (including the low- $x$ region, and including high- $E_{T, B}^{2}$, low- $Q^{2}$ processes) could be obtained in a theoretically consistent manner, consistent with factorisation. To reduce the merging uncertainty, modifications to the parton shower kernels were made. Finite terms were added to the previously used dipole kernel to ensure that the kernels amount to the full matrix elements associated to the splitting process (like in antenna-based showers). With these refinements, SHERPA is the first multi-purpose event generator program for deepinelastic processes which incorporates a full merging of leading-order matrix elements with parton showers.

We validated our results on a multitude of HERA data on hadronic final states in deep-inelastic scattering, including jet cross sections, jet-transition rates and hadronic particle spectra. All observables considered are described in a very satisfactory manner. We quantified the uncertainties due to scale choices, merging parameters, parton-shower schemes, parton distribution functions and hadronisation models.

The comparison with HERA data provides an important validation of the SHERPA initial- and final-state parton-shower schemes in non-trivial kinematical situations hardly accessible in other collider environments. It has important consequences for LHC studies in similar kinematical situations (like low-mass Drell-Yan or vector-boson plus multi-jet production).

Using the HERA data set on different aspects of hadronic final states will allow for a validation and tuning of hadronisation models, which was based up to now purely on data from $e^{+} e^{-}$annihilation. Inclusion of DIS data probes different flavour combinations, and will help to resolve parameter degeneracies, thereby leading to important improvements of the hadronisation models.

\section{Acknowledgements}

We thank Hendrik Hoeth for providing a dedicated tune of the Lund string fragmentation model in the implementation of Pyhtia 6.4.18 and Eike von Seggern for providing a tune of the cluster fragmentation model in SHERPA. We gratefully acknowledge discussions with Alberto Guffanti, Frank Krauss, Steffen Schumann and Frank Siegert.

This work was funded in part by the Swiss National Science Foundation (SNF, contract number 200020126691) and by the University of Zürich (Forschungskredit number 57183003).

\section{A Matrix element correction of the splitting kernels}

The original parton shower algorithm [22], based on Catani-Seymour dipoles, can be modified easily to improve the radiation pattern in deep-inelastic scattering. The key idea is to add some nonsingular bits to the original spin-averaged dipole functions, such that combining the radiation functions of emitter and spectator yields the exact NLO real radiation matrix element. This correction does not spoil the logarithmic accuracy of the parton shower. However, merging the shower with higher-order tree-level matrix elements along the lines of Sec. 2.2 is then alleviated for the first emission, because the radiation patterns are formally identical. ${ }^{3}$ We focus on massless partons.

The situation in final-state parton splittings with initial-state spectator is sketched in Fig. 4b. We employ the variables

$$
\tilde{z}_{i}=\frac{p_{i} p_{a}}{\left(p_{i}+p_{j}\right) p_{a}} \quad \text { and } \quad x_{i j, a}=1-\frac{p_{i} p_{j}}{\left(p_{i}+p_{j}\right) p_{a}}
$$

The corresponding spin-averaged splitting functions are given in [22]. While $\left\langle\mathbf{V}_{g^{i} g^{j}}^{a}\right\rangle$ is left unchanged, we

\footnotetext{
${ }^{3}$ In fact the identity of radiation patterns largely depends on the recoil scheme of the parton shower, cf. [65]. In this context, we only refer to the processes $\gamma^{*} g \rightarrow q \bar{q}$ and $\gamma^{*} q \rightarrow q g$, averaged over the virtual photon spin.
} 


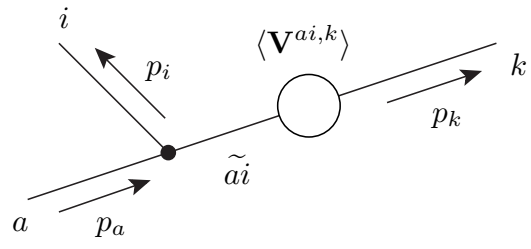

(a)

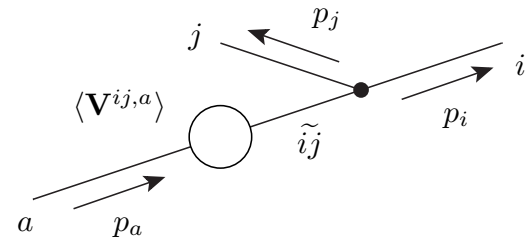

(b)

Figure 5: Schematic view of the splittings of an initial-state parton with a finalstate spectator and the splitting of a final-state parton with an initialstate spectator. The blob denotes the hard matrix element. Incoming and outgoing lines label initial- and final-state partons, respectively.

redefine, in the context of this work

$$
\begin{aligned}
& \left\langle\mathbf{V}_{q_{i} g_{j}}^{a}\right\rangle\left(\tilde{z}_{i}, x_{i j, a}\right)=C_{F}\left\{\frac{2}{1-\tilde{z}_{i}+\left(1-x_{i j, a}\right)}-\left(1+\tilde{z}_{i}\right)+C_{q_{i} g_{j}}^{\mathrm{DIS}}\left(\tilde{z}_{i}, x_{i j, a}\right)\right\}, \\
& \left\langle\mathbf{V}_{q_{i} q_{j}}^{a}\right\rangle\left(\tilde{z}_{i}, x_{i j, a}\right)=T_{R}\left\{\left(1-2 \tilde{z}_{i}\left(1-\tilde{z}_{i}\right)\right)\left(1-\frac{C_{q_{i} q_{j}}^{\mathrm{DIS}}\left(x_{i j, a}, \tilde{z}_{i}\right)}{2\left(1-\tilde{z}_{i}\right)}\right)+C_{q_{i} q_{j}}^{\mathrm{DIS}}\left(\tilde{z}_{i}, x_{i j, a}\right)\right\} .
\end{aligned}
$$

These functions differ from the original evolution kernels by the additional nonsingular factors

$$
\begin{aligned}
& C_{q_{i} g_{j}}^{\mathrm{DIS}}\left(\tilde{z}_{i}, x_{i j, a}\right)=\left(1-x_{i j, a}\right)\left[1+3 x_{i j, a} \tilde{z}_{i}\right], \\
& C_{q_{i} q_{j}}^{\mathrm{DIS}}\left(\tilde{z}_{i}, x_{i j, a}\right)=4\left(1-x_{i j, a}\right) \tilde{z}_{i}\left(1-\tilde{z}_{i}\right) .
\end{aligned}
$$

As required, the corrections vanish in the soft and collinear limits and the original evolution kernels remain. An initial-state parton splitting with final-state spectator is sketched in Fig. 4a. We employ the variables

$$
u_{i}=\frac{p_{i} p_{a}}{\left(p_{i}+p_{k}\right) p_{a}} \quad \text { and } \quad x_{i k, a}=1-\frac{p_{i} p_{k}}{\left(p_{i}+p_{k}\right) p_{a}} .
$$

The corresponding spin-averaged splitting functions $\langle\mathbf{V}\rangle$ are presented in [22]. While $\left\langle\mathbf{V}_{k}^{q_{a} q_{i}}\right\rangle$ and $\left\langle\mathbf{V}_{k}^{g_{a} g_{i}}\right\rangle$ are left unchanged, we redefine, in the context of this work

$$
\begin{aligned}
& \left\langle\mathbf{V}_{k}^{q_{a} g_{i}}\right\rangle\left(x_{i k, a}, u_{i}\right)=C_{F}\left\{\frac{2}{1-x_{i k, a}+u_{i}}-\left(1+x_{i k, a}\right)+C_{q_{a} g_{i}}^{\mathrm{DIS}}\left(x_{i k, a}, u_{k}\right)\right\}, \\
& \left\langle\mathbf{V}_{k}^{g_{a} q_{i}}\right\rangle\left(x_{i k, a}, u_{i}\right)=T_{R}\left\{\left(1-2 x_{i k, a}\left(1-x_{i k, a}\right)\right)\left(1-\frac{C_{q_{a i} q_{i}}^{\mathrm{DIS}}\left(u_{k}, x_{i k, a}\right)}{2 x_{i k, a}}\right)+C_{q_{a i} q_{i}}^{\mathrm{DIS}}\left(x_{i k, a}, u_{k}\right)\right\} .
\end{aligned}
$$

Using the above modifications, it can be shown that the combination of appropriate splitting kernels indeed reproduces the complete real-emission matrix elements for the processes $\gamma^{*} g \rightarrow q \bar{q}$ and $\gamma^{*} q \rightarrow q g$.

\section{References}

[1] V. N. Gribov and L. N. Lipatov, Deep inelastic e-p scattering in perturbation theory, Sov. J. Nucl. Phys. 15 (1972), 438-450. L. N. Lipatov, The parton model and perturbation theory, Sov. J. Nucl. Phys. 20 (1975), 94-102. Y. L. Dokshitzer, Calculation of the structure functions for deep inelastic scattering and $e^{+} e^{-}$annihilation by perturbation theory in quantum chromodynamics, Sov. Phys. JETP 46 (1977), 641-653. G. Altarelli and G. Parisi, Asymptotic freedom in parton language, Nucl. Phys. B126 (1977), 298-318. 
[2] G. Curci, W. Furmanski and R. Petronzio, Evolution of parton densities beyond leading order: The nonsinglet case, Nucl. Phys. B175 (1980), 27. W. Furmanski and R. Petronzio, Singlet parton densities beyond leading order, Phys. Lett. B97 (1980), 437. S. Moch, J. A. M. Vermaseren and A. Vogt, The three-loop splitting functions in QCD: The non-singlet case, Nucl. Phys. B688 (2004), 101-134, [hep-ph/0403192]. A. Vogt, S. Moch and J. A. M. Vermaseren, The three-loop splitting functions in QCD: The singlet case, Nucl. Phys. B691 (2004), 129-181, [hep-ph/0404111].

[3] A. D. Martin, W. J. Stirling, R. S. Thorne and G. Watt, Parton distributions for the LHC, Eur. Phys. J. C63 (2009), 189-295, [arXiv:0901.0002 [hep-ph]].

[4] R. D. Ball et al., NNPDF collaboration, A determination of parton distributions with faithful uncertainty estimation, Nucl. Phys. B809 (2009), 1-63, [arXiv:0808.1231 [hep-ph]].

[5] P. M. Nadolsky et al., Implications of CTEQ global analysis for collider observables, Phys. Rev. D78 (2008), 013004, [arXiv:0802.0007 [hep-ph]].

[6] P. Jimenez-Delgado and E. Reya, Dynamical NNLO parton distributions, Phys. Rev. D79 (2009), 074023, [arXiv:0810.4274 [hep-ph]].

[7] E. A. Kuraev, L. N. Lipatov and V. S. Fadin, The Pomeranchuk Singularity in Nonabelian Gauge Theories, Sov. Phys. JETP 45 (1977), 199-204. I. I. Balitsky and L. N. Lipatov, The Pomeranchuk Singularity in Quantum Chromodynamics, Sov. J. Nucl. Phys. 28 (1978), 822-829.

[8] M. Ciafaloni, Coherence effects in initial jets at small $Q^{2} / s$, Nucl. Phys. B296 (1988), 49-74. S. Catani, F. Fiorani and G. Marchesini, QCD coherence in initial state radiation, Phys. Lett. B234 (1990), 339-345. S. Catani, F. Fiorani and G. Marchesini, Small-x behavior of initial state radiation in perturbative QCD, Nucl. Phys. B336 (1990), 18-85. G. Marchesini, QCD coherence in the structure function and associated distributions at small x, Nucl. Phys. B445 (1995), 49-80, [hep-ph/9412327].

[9] A. Aktas et al., H1 collaboration, Forward jet production in deep inelastic scattering at HERA, Eur. Phys. J. C46 (2006), 27-42, [hep-ex/0508055], DESY-05-135. S. Chekanov et al., ZEUS collaboration, Forward-jet production in deep inelastic ep scattering at HERA, Eur. Phys. J. C52 (2007), 515-530, [arXiv:0707.3093 [hep-ex]].

[10] E. Mirkes and D. Zeppenfeld, Dijet production at HERA in next-to-leading order, Phys. Lett. B380 (1996), 205-212, [hep-ph/9511448]. S. Catani and M. H. Seymour, The Dipole Formalism for the Calculation of QCD Jet Cross Sections at Next-to-Leading Order, Phys. Lett. B378 (1996), 287-301, [hep-ph/9602277].

[11] Z. Nagy and Z. Trocsanyi, Multi Jet Cross Sections in Deep Inelastic Scattering at Next-To-Leading Order, Phys. Rev. Lett. 87 (2001), 082001, [hep-ph/0104315]. Z. Nagy and Z. Trocsanyi, Three-jet event-shapes in lepton-proton scattering at next-to-leading order accuracy, Phys. Lett. B634 (2006), 498-503, [hep-ph/0511328].

[12] A. Aktas et al., H1 collaboration, Measurement of inclusive jet production in deep-inelastic scattering at high $Q^{2}$ and determination of the strong coupling, Phys. Lett. B653 (2007), 134-144, [arXiv:0706.3722 [hep-ex]], DESY-07-073. S. Chekanov et al., ZEUS collaboration, Inclusive-jet and dijet cross sections in deep inelastic scattering at HERA, Nucl. Phys. B765 (2007), 1-30, [hep-ex/0608048].

[13] G. A. Schuler and T. Sjöstrand, A scenario for high-energy $\gamma \gamma$ interactions, Z. Phys. C73 (1997), 677-688, [hep-ph/9605240].

[14] B. Pötter, JetViP 1.1: Calculating one jet and two jet cross-sections with virtual photons in NLO QCD, Comput. Phys. Commun. 119 (1999), 45-66, [hep-ph/9806437].

[15] G. Kramer and B. Pötter, Forward jet production at small $x$ in next-to-leading order QCD, Phys. Lett. B453 (1999), 295-301, [hep-ph/9901314].

[16] M. Bengtsson and T. Sjöstrand, A comparative study of coherent and non-coherent parton shower evolution, Nucl. Phys. B289 (1987), 810. T. Sjöstrand, A model for initial state parton showers, Phys. Lett. B157 (1985), 321. 
[17] G. Marchesini and B. R. Webber, Simulation of QCD Jets Including Soft Gluon Interference, Nucl. Phys. B238 (1984), 1. G. Marchesini and B. R. Webber, Monte Carlo Simulation of General Hard Processes with Coherent QCD Radiation, Nucl. Phys. B310 (1988), 461.

[18] T. Sjöstrand and P. Z. Skands, Transverse-momentum-ordered showers and interleaved multiple interactions, Eur. Phys. J. C39 (2005), 129-154, [hep-ph/0408302].

[19] R. Kuhn, F. Krauss, B. Ivanyi and G. Soff, APACIC++ 1.0: A PArton Cascade In C++, Comput. Phys. Commun. 134 (2001), 223-266, [hep-ph/0004270]. F. Krauss, A. Schälicke and G. Soff, APACIC++ 2.0: A PArton Cascade In $C++$, Comput. Phys. Commun. 174 (2006), 876-902, [hep-ph/0503087].

[20] G. Marchesini and B. R. Webber, Simulation of QCD initial state radiation at small $x$, Nucl. Phys. B349 (1991), 617-634. G. Marchesini and B. R. Webber, Final states in heavy quark leptoproduction at small $x$, Nucl. Phys. B386 (1992), 215-235. H. Jung, The CCFM Monte Carlo generator CASCADE, Comput. Phys. Commun. 143 (2002), 100-111, [hep-ph/0109102]. K. Golec-Biernat, S. Jadach, W. Płaczek, P. Stephens and M. Skrzypek, Markovian Monte Carlo solutions of the oneloop CCFM equations, Acta Phys. Polon. B38 (2007), 3149-3168, [hep-ph/0703317].

[21] L. Lönnblad, Ariadne version 4: A program for simulation of QCD cascades implementing the colour dipole model, Comput. Phys. Commun. 71 (1992), 15-31. J.-C. Winter and F. Krauss, Initialstate showering based on colour dipoles connected to incoming parton lines, JHEP 07 (2008), 040, [arXiv:0712.3913 [hep-ph]].

[22] S. Schumann and F. Krauss, A parton shower algorithm based on Catani-Seymour dipole factorisation, JHEP 03 (2008), 038, [arXiv:0709.1027 [hep-ph]].

[23] M. Dinsdale, M. Ternick and S. Weinzierl, Parton showers from the dipole formalism, Phys. Rev. D76 (2007), 094003, [arXiv:0709.1026 [hep-ph]].

[24] T. Gleisberg, F. Krauss, A. Schälicke, S. Schumann and J.-C. Winter, Studying $W^{+} W^{-}$production at the Fermilab Tevatron with SHERPA, Phys. Rev. D72 (2005), 034028, [hep-ph/0504032]. J. Alwall et al., Comparative study of various algorithms for the merging of parton showers and matrix elements in hadronic collisions, Eur. Phys. J. C53 (2008), 473-500, [arXiv:0706. 2569 [hep-ph]].

[25] M. L. Mangano, M. Moretti, F. Piccinini and M. Treccani, Matching matrix elements and shower evolution for top-pair production in hadronic collisions, JHEP 01 (2007), 013, [hep-ph/0611129]. J. Alwall, S. de Visscher and F. Maltoni, QCD radiation in the production of heavy colored particles at the LHC, JHEP 02 (2009), 017, [arXiv:0810.5350 [hep-ph]].

[26] S. Catani, F. Krauss, R. Kuhn and B. R. Webber, QCD matrix elements + parton showers, JHEP 11 (2001), 063, [hep-ph/0109231]. F. Krauss, Matrix elements and parton showers in hadronic interactions, JHEP 0208 (2002), 015, [hep-ph/0205283].

[27] M. L. Mangano, M. Moretti and R. Pittau, Multijet matrix elements and shower evolution in hadronic collisions: $W b \bar{b}+n$-jets as a case study, Nucl. Phys. B632 (2002), 343-362, [hep-ph/0108069].

[28] S. Höche, F. Krauss, S. Schumann and F. Siegert, QCD matrix elements and truncated showers, JHEP 05 (2009), 053, [arXiv:0903.1219 [hep-ph]].

[29] K. Hamilton, P. Richardson and J. Tully, A modified CKKW matrix element merging approach to angular-ordered parton showers, JHEP 11 (2009), 038, [arXiv:0905. 3072 [hep-ph]].

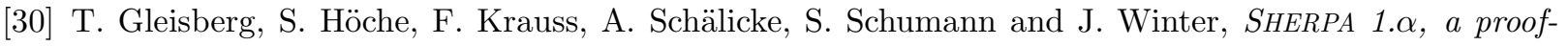
of-concept version, JHEP 02 (2004), 056, [hep-ph/0311263]. T. Gleisberg, S. Höche, F. Krauss, M. Schönherr, S. Schumann, F. Siegert and J. Winter, Event generation with SHERPA 1.1, JHEP 02 (2009), 007, [arXiv:0811.4622 [hep-ph]].

[31] B. Andersson, G. Gustafson, G. Ingelman and T. Sjöstrand, Parton Fragmentation and String Dynamics, Phys. Rept. 97 (1983), 31-145. B. Andersson, The Lund model, vol. 7, Camb. Monogr. Part. Phys. Nucl. Phys. Cosmol., 1997. 
[32] R. D. Field and S. Wolfram, A QCD model for $e^{+} e^{-}$annihilation, Nucl. Phys. B213 (1983), 65. T. D. Gottschalk, An improved description of hadronization in the QCD cluster model for $e^{+} e^{-}$annihilation, Nucl. Phys. B239 (1984), 349. B. R. Webber, A QCD model for jet fragmentation including soft gluon interference, Nucl. Phys. B238 (1984), 492.

[33] SHERPA's new hadronisation model, in preparation. J.-C. Winter, F. Krauss and G. Soff, A modified cluster-hadronisation model, Eur. Phys. J. C36 (2004), 381-395, [hep-ph/0311085].

[34] T. Plehn, D. Rainwater and P. Skands, Squark and gluino production with jets, Phys. Lett. B645 (2007), 217-221, [hep-ph/0510144].

[35] Y. L. Dokshitzer, G. Marchesini and G. P. Salam, Revisiting parton evolution and the large-x limit, Phys. Lett. B634 (2006), 504-507, [hep-ph/0511302]. Y. L. Dokshitzer, A new look for good old parton dynamics, arXiv:0911.0847 [hep-ph].

[36] S. Catani and M. H. Seymour, A general algorithm for calculating jet cross sections in NLO QCD, Nucl. Phys. B485 (1997), 291-419, [hep-ph/9605323]. S. Catani, S. Dittmaier, M. H. Seymour and Z. Trocsanyi, The dipole formalism for next-to-leading order QCD calculations with massive partons, Nucl. Phys. B627 (2002), 189-265, [hep-ph/0201036].

[37] S. Plätzer and S. Gieseke, Coherent Parton Showers with Local Recoils, arXiv:0909.5593 [hep-ph].

[38] S. Höche, S. Schumann and F. Siegert, Hard photon production and matrix-element parton-shower merging, arXiv:0912.3501 [hep-ph].

[39] S. Catani, Y. L. Dokshitzer and B. R. Webber, The $k_{\perp}$ clustering algorithm for jets in deep inelastic scattering and hadron collisions, Phys. Lett. B285 (1992), 291-299.

[40] A. Gehrmann-De Ridder, T. Gehrmann and E. W. N. Glover, Antenna subtraction at NNLO, JHEP 09 (2005), 056, [hep-ph/0505111]. A. Daleo, T. Gehrmann and D. Maitre, Antenna subtraction with hadronic initial states, JHEP 04 (2007), 016, [hep-ph/0612257].

[41] W. T. Giele, D. A. Kosower and P. Z. Skands, A Simple shower and matching algorithm, Phys. Rev. D78 (2008), 014026, [arXiv:0707.3652 [hep-ph]].

[42] L. Lönnblad, Correcting the colour-dipole cascade model with fixed order matrix elements, JHEP 05 (2002), 046, [hep-ph/0112284]. N. Lavesson and L. Lönnblad, Extending CKKW-merging to one-loop matrix elements, JHEP 12 (2008), 070, [arXiv:0811.2912 [hep-ph]].

[43] F. Krauss, A. Schälicke, S. Schumann and G. Soff, Simulating $W / Z+$ jets production at the Tevatron, Phys. Rev. D70 (2004), 114009, [hep-ph/0409106]. A. Schälicke and F. Krauss, Implementing the ME+PS merging algorithm, JHEP 07 (2005), 018, [hep-ph/0503281].

[44] N. Brook, R. G. Waugh, T. Carli, R. Mohr and M. Sutton, Tuning Monte Carlo event generators to HERA data, In Hamburg 1995/96, Future physics at HERA, vol. 1, 613-619. N. H. Brook et al., A Comparison of Deep Inelastic Scattering Monte Carlo Event Generators to HERA Data, hep-ex/9912053, In Hamburg 1998/1999, Monte Carlo generators for HERA physics, 10-35.

[45] T. Gleisberg and S. Höche, Comix, a new matrix element generator, JHEP 12 (2008), 039, [arXiv:0808.3674 [hep-ph]].

[46] T. Sjöstrand, S. Mrenna and P. Skands, PYTHIA 6.4 physics and manual, JHEP 05 (2006), 026, [hep-ph/0603175].

[47] A. Buckley, H. Hoeth, H. Lacker, H. Schulz and J. E. von Seggern, Systematic event generator tuning for the $L H C$, arXiv:0907.2973 [hep-ph].

[48] F. Krauss, T. Laubrich and F. Siegert, Simulation of hadron decays in SHERPA, in preparation.

[49] M. Schönherr and F. Krauss, Soft photon radiation in particle decays in SHERPA, JHEP 12 (2008), 018, [arXiv:0810.5071 [hep-ph]].

[50] B. M. Waugh et al., HZTool and Rivet: Toolkit and framework for the comparison of simulated final states and data at colliders, hep-ph/0605034. 
[51] C. Adloff et al., H1 collaboration, Measurement of inclusive jet cross-sections in deep-inelastic ep scattering at HERA, Phys. Lett. B542 (2002), 193-206, [hep-ex/0206029], DESY-02-079.

[52] S. D. Ellis and D. E. Soper, Successive combination jet algorithm for hadron collisions, Phys. Rev. D48 (1993), 3160-3166, [hep-ph/9305266]. S. Catani, Y. L. Dokshitzer, M. H. Seymour and B. R. Webber, Longitudinally-invariant $k_{\perp}$-clustering algorithms for hadron-hadron collisions, Nucl. Phys. B406 (1993), 187-224.

[53] C. Adloff et al., H1 collaboration, Measurement and QCD analysis of jet cross sections in deep-inelastic positron-proton collisions at $\sqrt{s}$ of $300 \mathrm{GeV}$, Eur. Phys. J. C19 (2001), 289-311, [hep-ex/0010054], DESY-00-145.

[54] S. Frixione and G. Ridolfi, Jet photoproduction at HERA, Nucl. Phys. B507 (1997), 315-333, [hep-ph/9707345].

[55] A. Aktas et al., H1 collaboration, Inclusive dijet production at low Bjørken-x in deep inelastic scattering, Eur. Phys. J. C33 (2004), 477-493, [hep-ex/0310019], DESY-03-160.

[56] C. Adloff et al., H1 collaboration, Three-jet production in deep-inelastic scattering at HERA, Phys. Lett. B515 (2001), 17-29, [hep-ex/0106078], DESY-01-073.

[57] S. Abachi et al., D0 collaboration, Studies of topological distributions of the three- and four-jet events in $\bar{p} p$ collisions at $\sqrt{s}=1800 \mathrm{GeV}$ with the Do detector, Phys. Rev. D53 (1996), 6000-6016, [hep-ex/9509005].

[58] C. Adloff et al., H1 collaboration, Measurement of internal jet structure in dijet production in deepinelastic scattering at HERA, Nucl. Phys. B545 (1999), 3-20, [hep-ex/9901010], DESY-98-210.

[59] L. A. Del Pozo, Tests of QCD from Jet and Hadron Production at LEP, PhD Thesis, University of Cambridge (1993), RALT-002. R. Akers et al., OPAL collaboration, QCD studies using a cone-based jet finding algorithm for $e^{+} e^{-}$collisions at LEP, Z. Phys. C63 (1994), 197-212.

[60] M. Kuhlen, Hadronic Final States in Deeply Inelastic Scattering, hep-ex/9508014, Proceedings of the workshop on DIS and QCD - DIS95, Paris, 1995 ed. J.F. Laporte and Y. Sirois. A. Edin, G. Ingelman and J. Rathsman, Soft colour interactions as the origin of rapidity gaps in DIS, Phys. Lett. B366 (1996), 371-378, [hep-ph/9508386]. A. Edin, G. Ingelman and J. Rathsman, Unified description of rapidity gaps and energy flows in DIS final states, Z. Phys. C75 (1997), 57-70, [hep-ph/9605281].

[61] C. Adloff et al., H1 collaboration, Measurements of transverse energy flow in deep-inelastic scattering at HERA, Eur. Phys. J. C12 (2000), 595-607, [hep-ex/9907027], DESY-99-091.

[62] C. Adloff et al., H1 collaboration, Measurement of charged particle transverse momentum spectra in deep inelastic scattering, Nucl. Phys. B485 (1997), 3-24, [hep-ex/9610006], DESY-96-215.

[63] M. Kuhlen, A new method to probe the low-x parton dynamics at HERA, Phys. Lett. B382 (1996), 441-446, [hep-ph/9606246]. M. Kuhlen, High-p $p_{T}$ particles in the forward region at HERA, hep-ex/9610004.

[64] S. Aid et al., H1 collaboration, Charged particle multiplicities in deep inelastic scattering at HERA, Z. Phys. C72 (1996), 573-592, [hep-ex/9608011], DESY-96-160.

[65] R. Kleiss, From two to three jets in heavy boson decays: An algorithmic approach, Phys. Lett. B180 (1986), 400. M. H. Seymour, A simple prescription for first-order corrections to quark scattering and annihilation processes, Nucl. Phys. B436 (1995), 443-460, [hep-ph/9410244].

[66] S. Alekhin, Parton distributions from deep-inelastic-scattering data, Phys. Rev. D68 (2003), 014002, [hep-ph/0211096].

[67] J. Pumplin, D. R. Stump, J. Huston, H. L. Lai, P. Nadolsky and W. K. Tung, New generation of parton distributions with uncertainties from global QCD analysis, JHEP 0207 (2002), 012, [hep-ph/0201195]. 


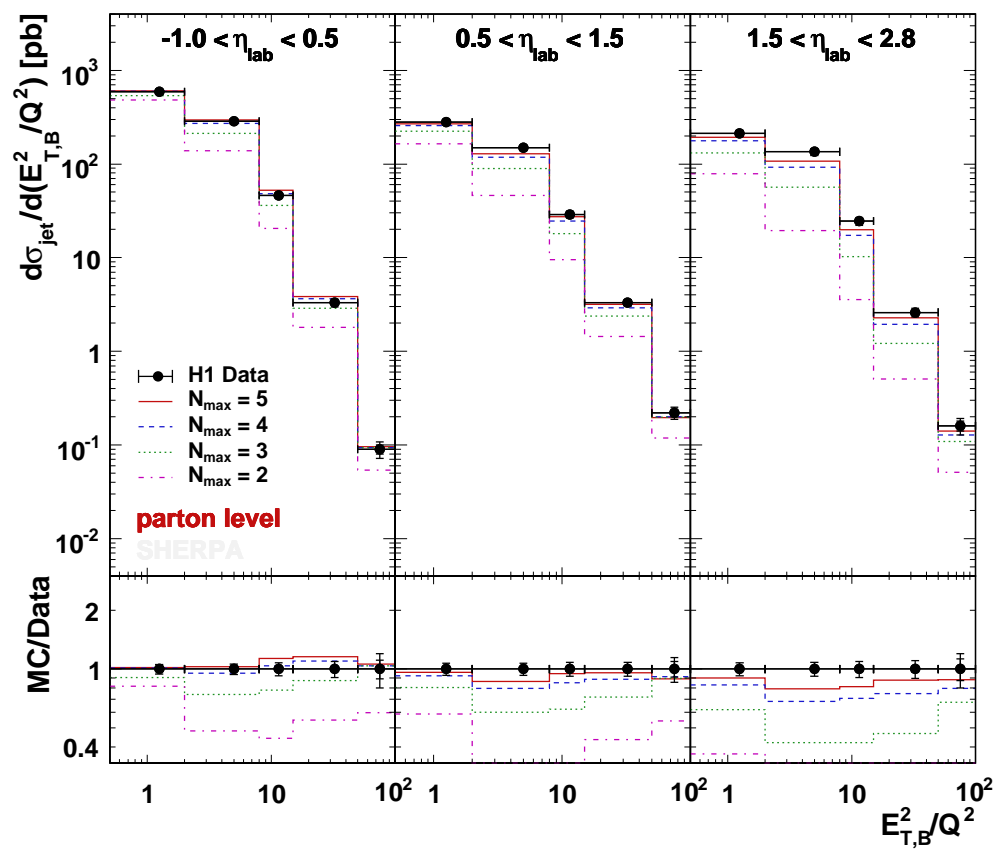

(a)

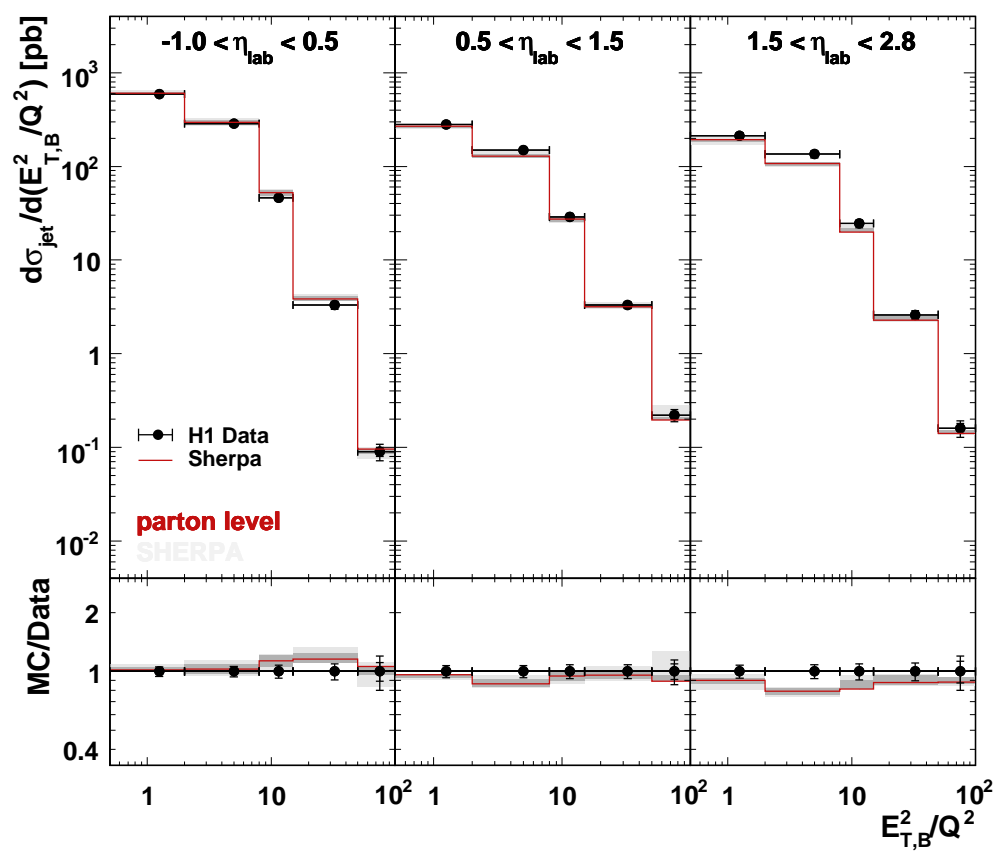

(b)

Figure 6: The inclusive jet cross section as a function of $E_{T, B}^{2} / Q^{2}$ in bins of $\eta_{l a b}$, measured by the H1 Collaboration [51]. $E_{T, B}^{2}$ is the jet transverse energy in the Breit frame, while $\eta_{l a b}$ denotes the jet rapidity in the laboratory frame. Part (a) displays the influence of the maximum parton multiplicity, $N_{\max }$, from hard matrix elements. We show the uncertainty originating from varying $S_{\text {DIS }}$ between 0.5 and 0.7 (light grey band) and from varying $\bar{Q}_{\text {cut }}$ between $3 \mathrm{GeV}$ and $9 \mathrm{GeV}$ (dark grey band) in part (b). 


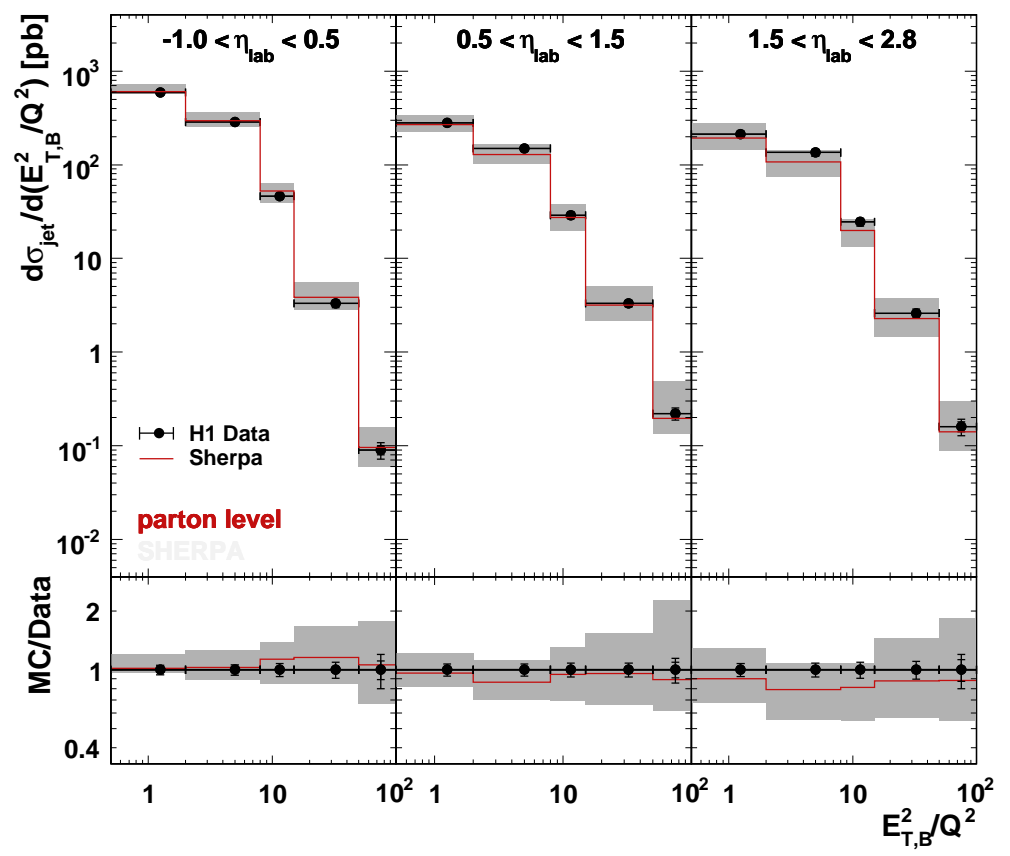

(a)

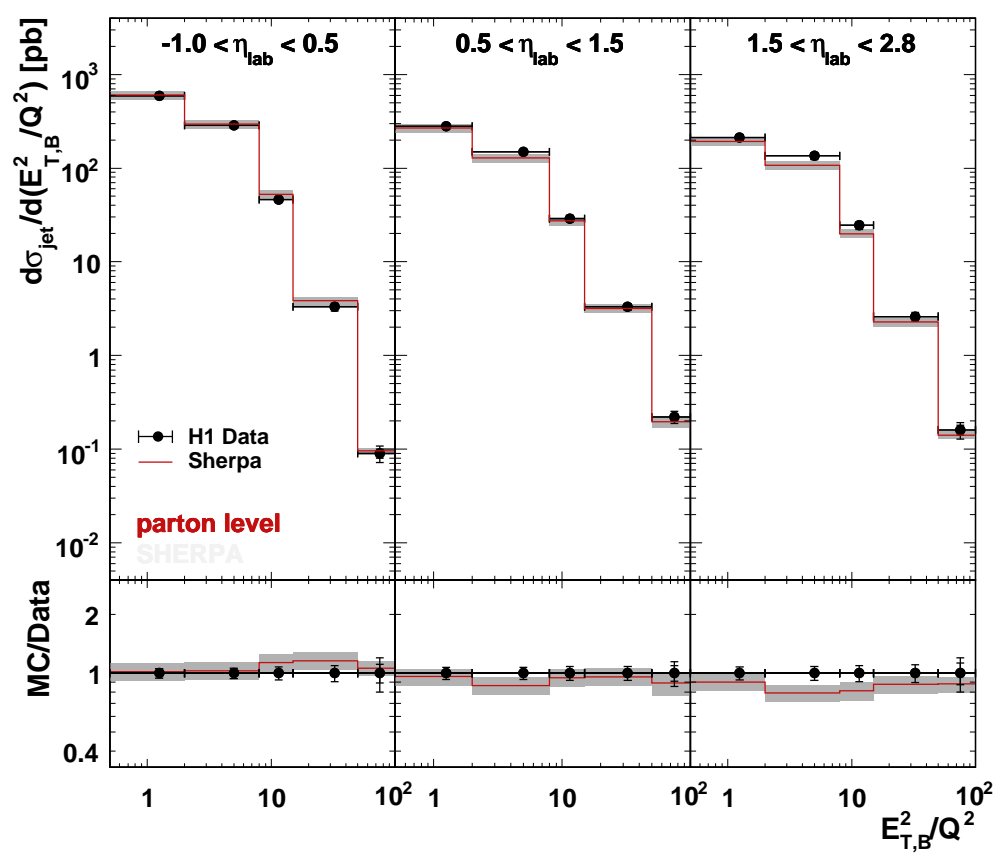

(b)

Figure 7: The inclusive jet cross section as a function of $E_{T, B}^{2} / Q^{2}$ in bins of $\eta_{l a b}$, measured by the H1 Collaboration [51], cf. Fig. 5. Part (a) presents the uncertainty associated with the variation of factorisation and renormalisation scales by factors of $1 / 2$ and 2. Part (b) shows the PDF uncertainty for the NNPDF 1.2 PDF set with 100 replicas [4]. 


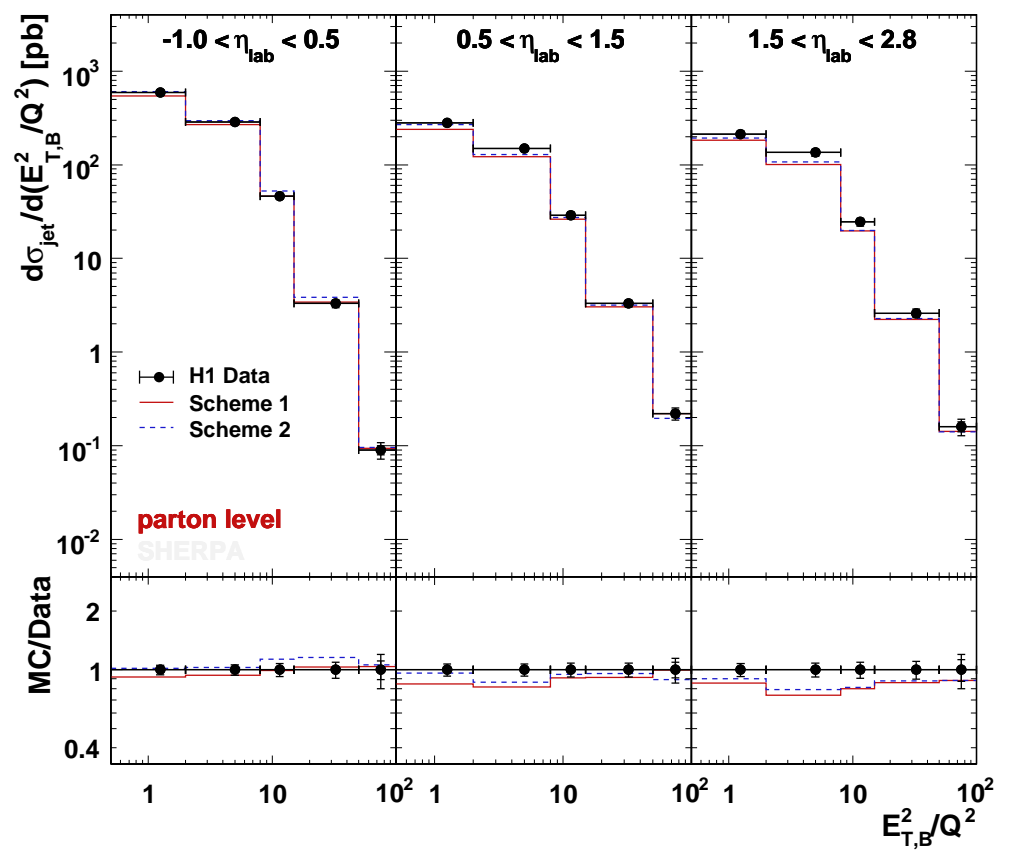

(a)

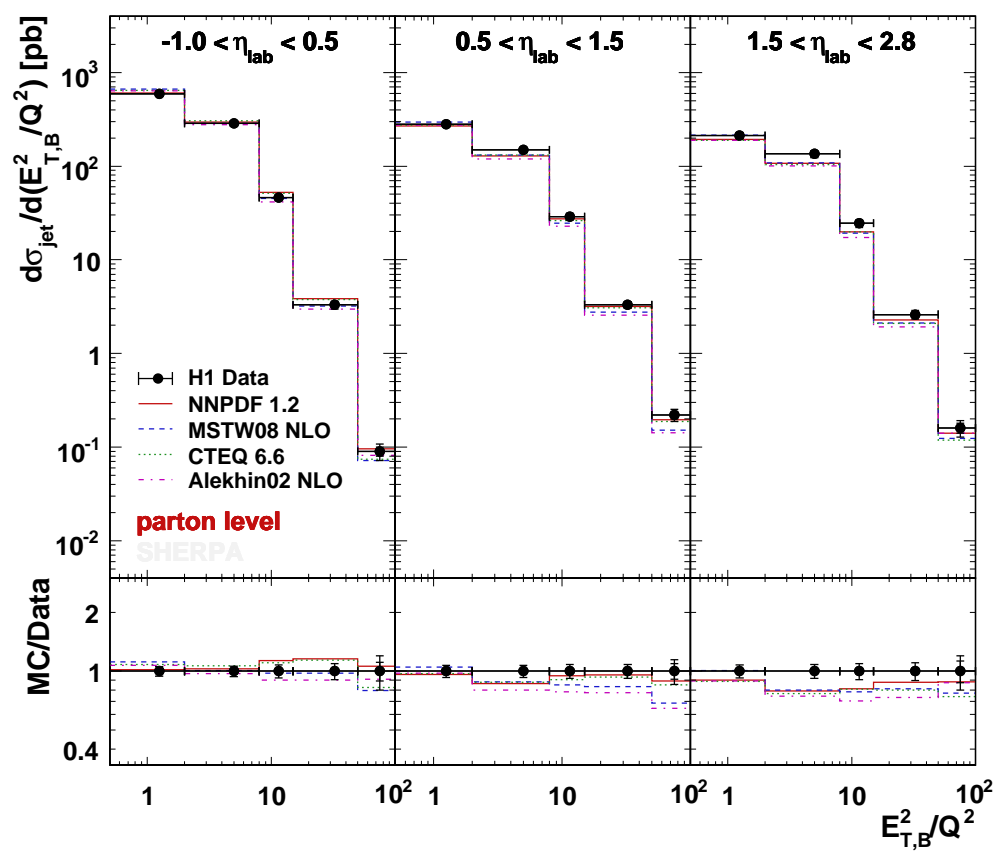

(b)

Figure 8: The inclusive jet cross section as a function of $E_{T, B}^{2} / Q^{2}$ in bins of $\eta_{l a b}$, measured by the H1 Collaboration [51], cf. Fig. 5. Part (a) displays the results for the two different parton-shower recoil strategies discussed in Sec. 2.1, cf. Fig. 1. Part (b) shows results obtained with the NLO PDF sets NNPDF 1.2 [4], MSTW 2008 [3], CTEQ 6.6 [5] and Alekhin 2002 [66]. 


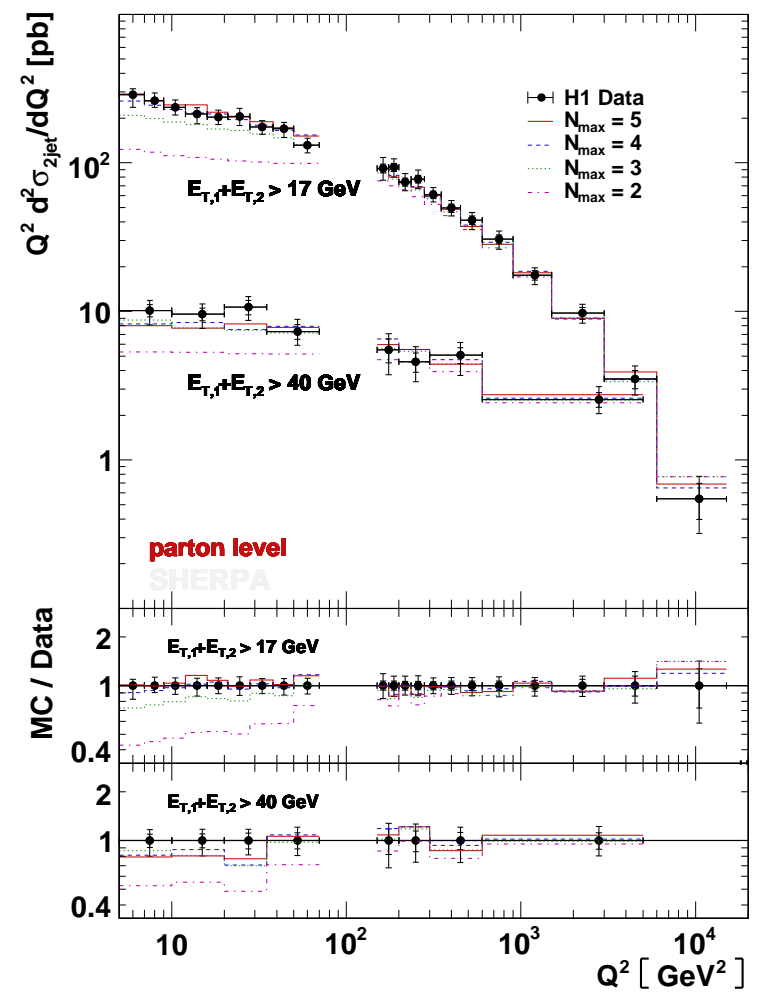

(a)

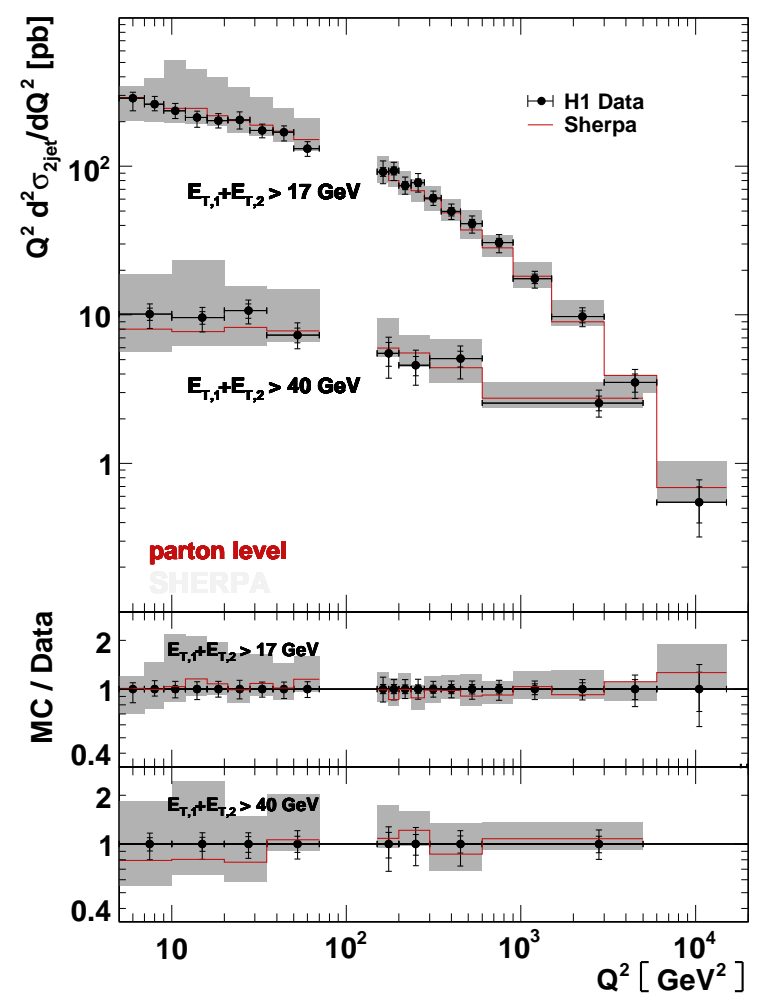

(c)

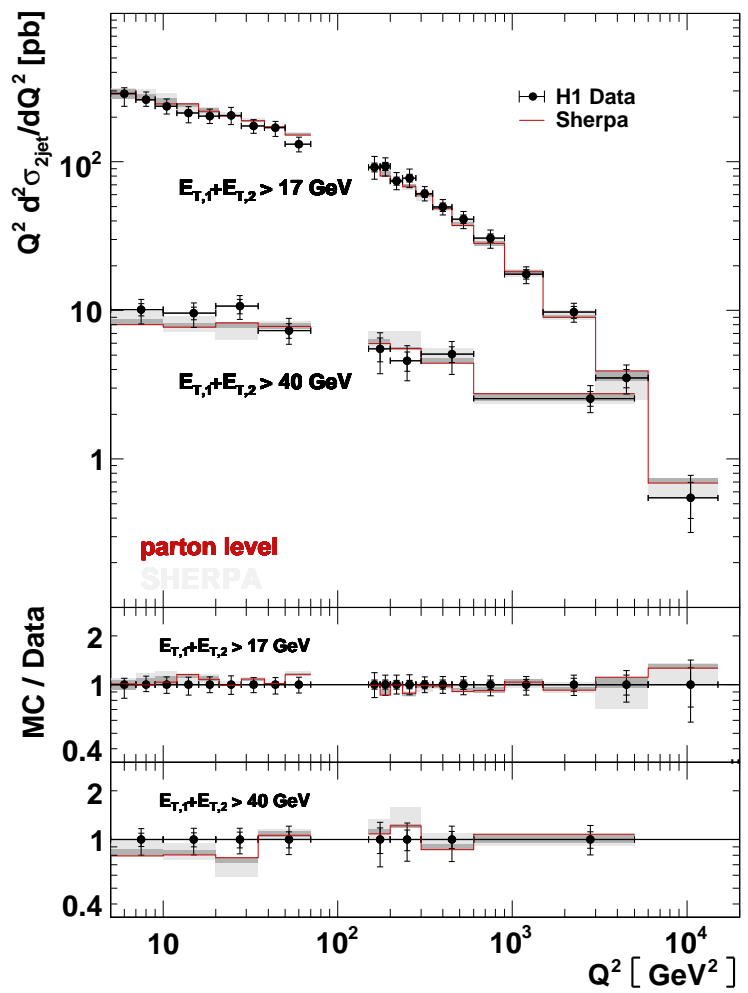

(b)

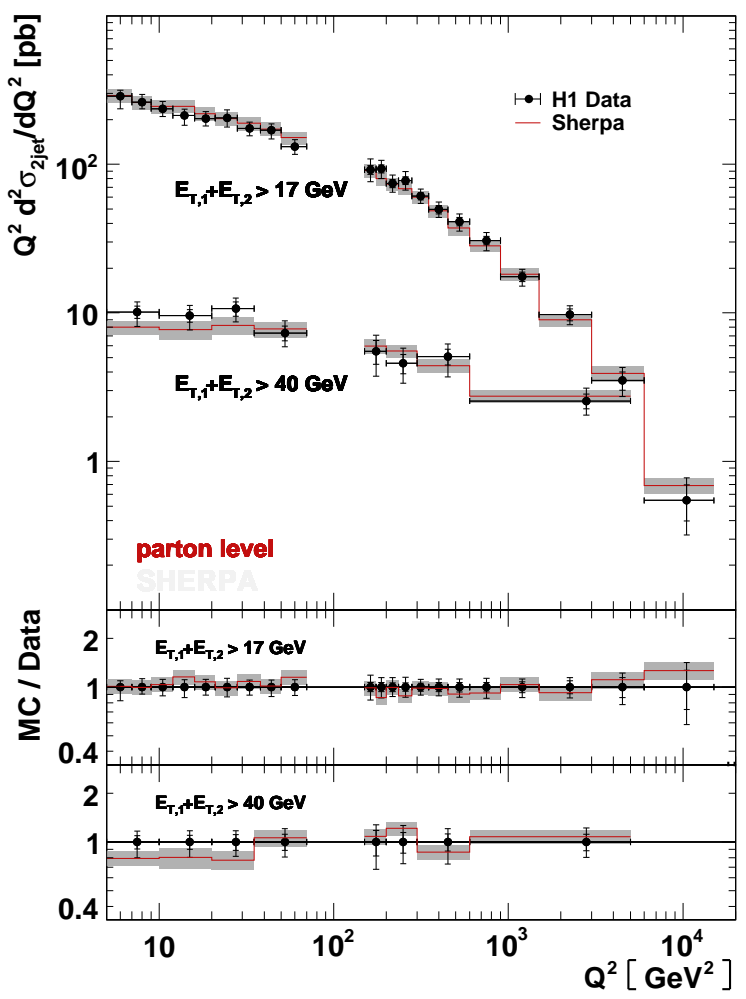

(d)

Figure 9: The di-jet cross section as a function of $Q^{2}$ in bins of $E_{T, 1}+E_{T, 2}$, measured by the H1 Collaboration [53]. See Figs. 5 and 6 for an explanation of parts (a) through (d). 


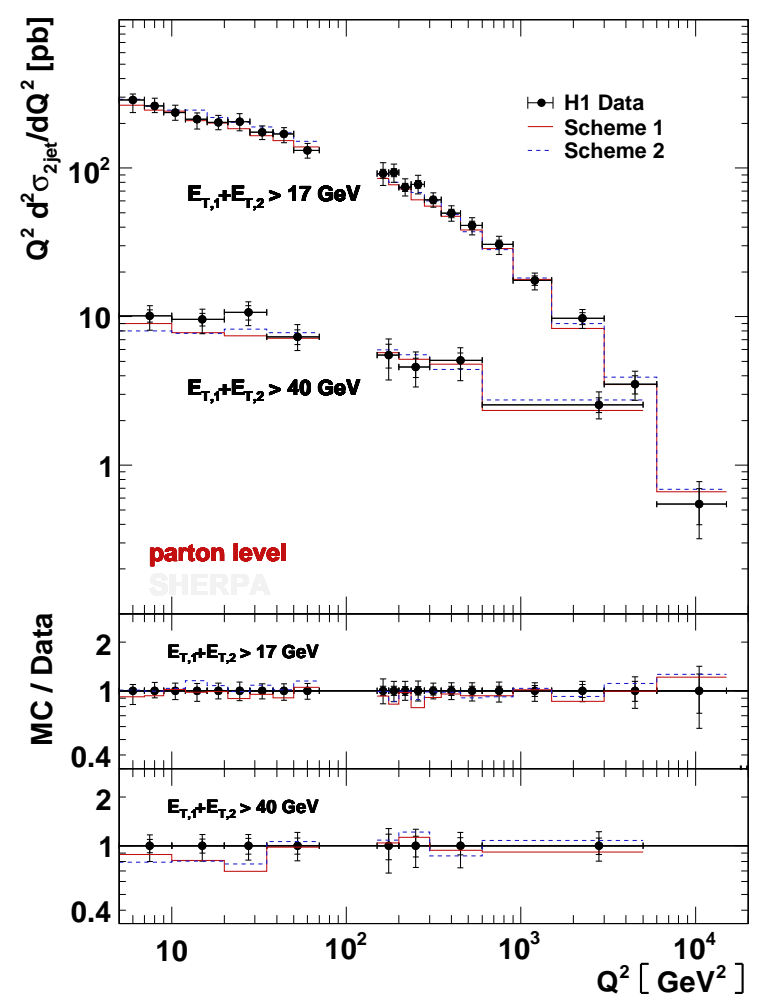

(a)

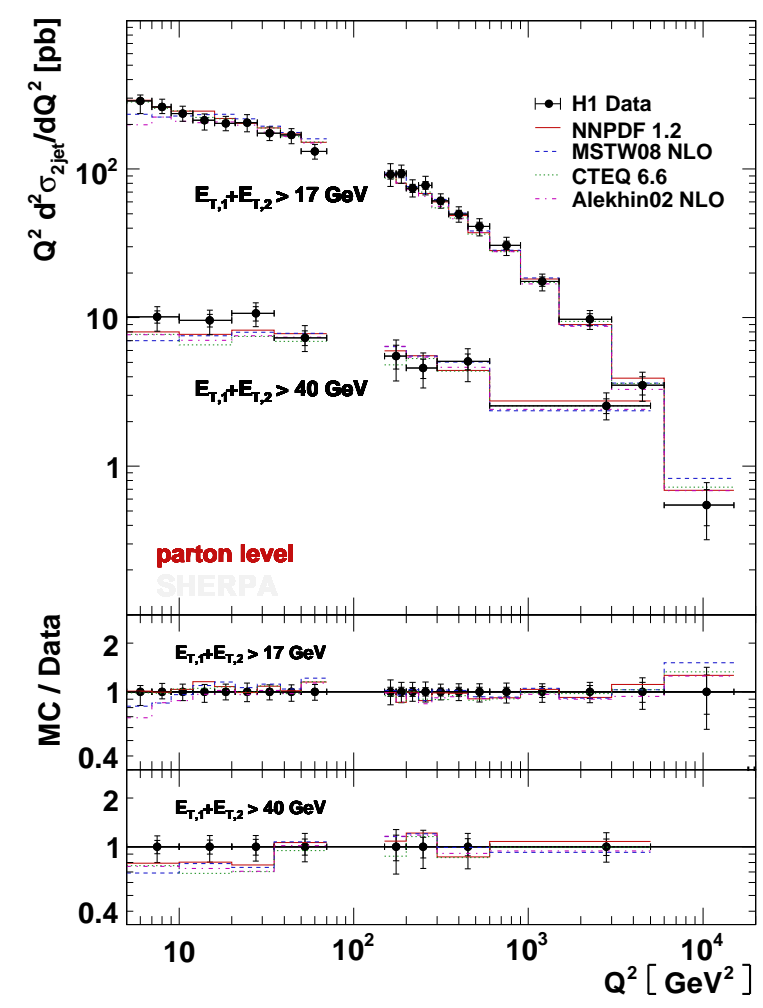

(b)

Figure 10: The di-jet cross section as a function of $Q^{2}$ in bins of $E_{T, 1}+E_{T, 2}$, measured by the H1 Collaboration [53]. See Fig. 7 for an explanation of parts (a) and (b). 

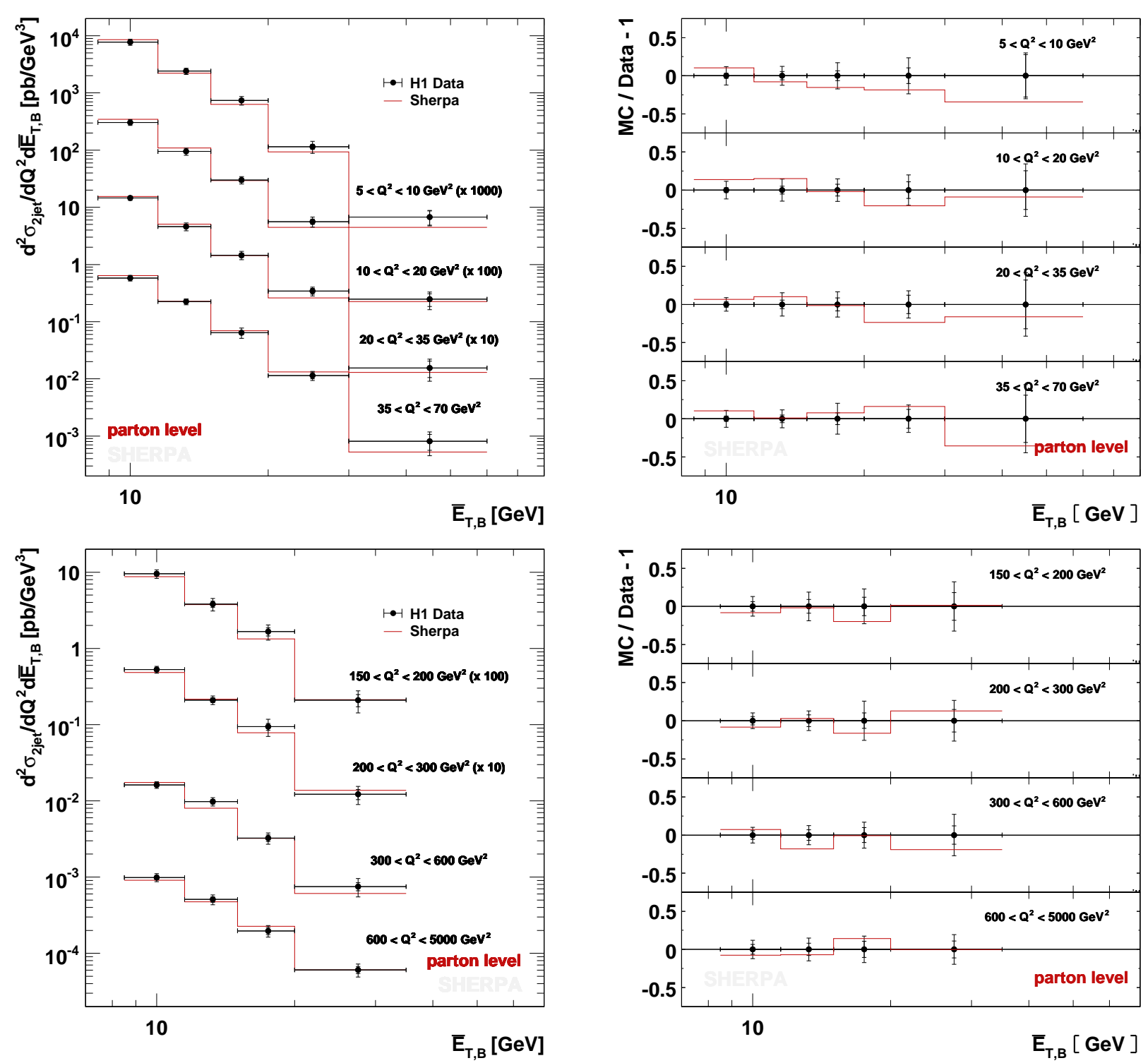

Figure 11: The di-jet cross section as a function of $\bar{E}_{T, B}$, the mean jet transverse energy in the Breit frame, measured by the H1 Collaboration [53]. 



Figure 12: The di-jet cross section as a function of $\eta^{\prime}$, measured by the H1 Collaboration [53]. $\eta^{\prime}$ denotes half the rapidity difference of the two leading jets in the Breit frame. 


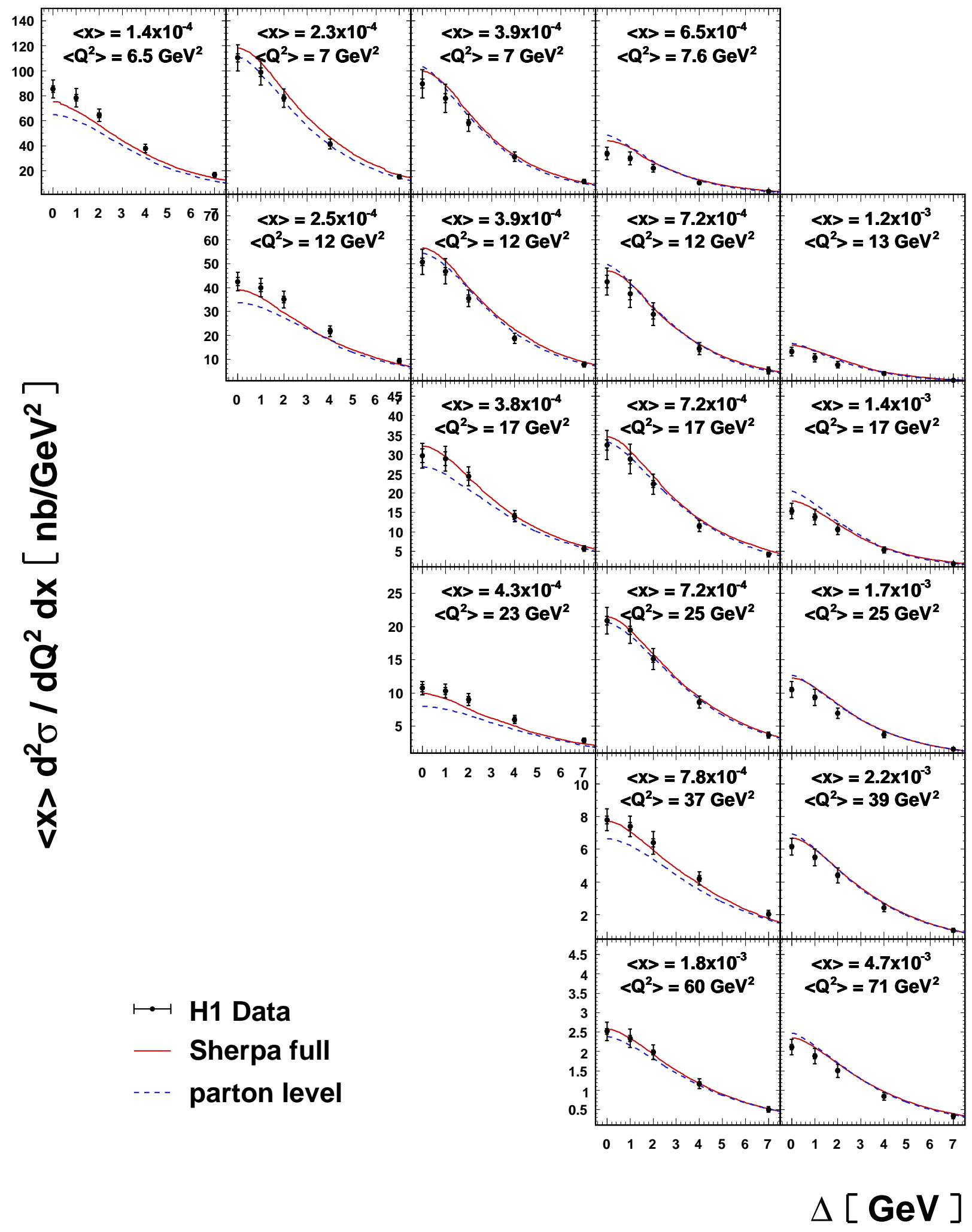

Figure 13: The differential di-jet cross section as a function of $\Delta$ in bins of mean $x$ and $Q^{2}$, measured by the H1 Collaboration [55]. $\Delta$ is defined as $E_{T, \max }^{*}>E_{T \text { cut }}^{*}+\Delta$, where $E_{T \text { cut }}^{*}$ is the minimum jet transverse energy and $E_{T \text { max }}^{*}$ is the transverse energy of the hardest jet. 

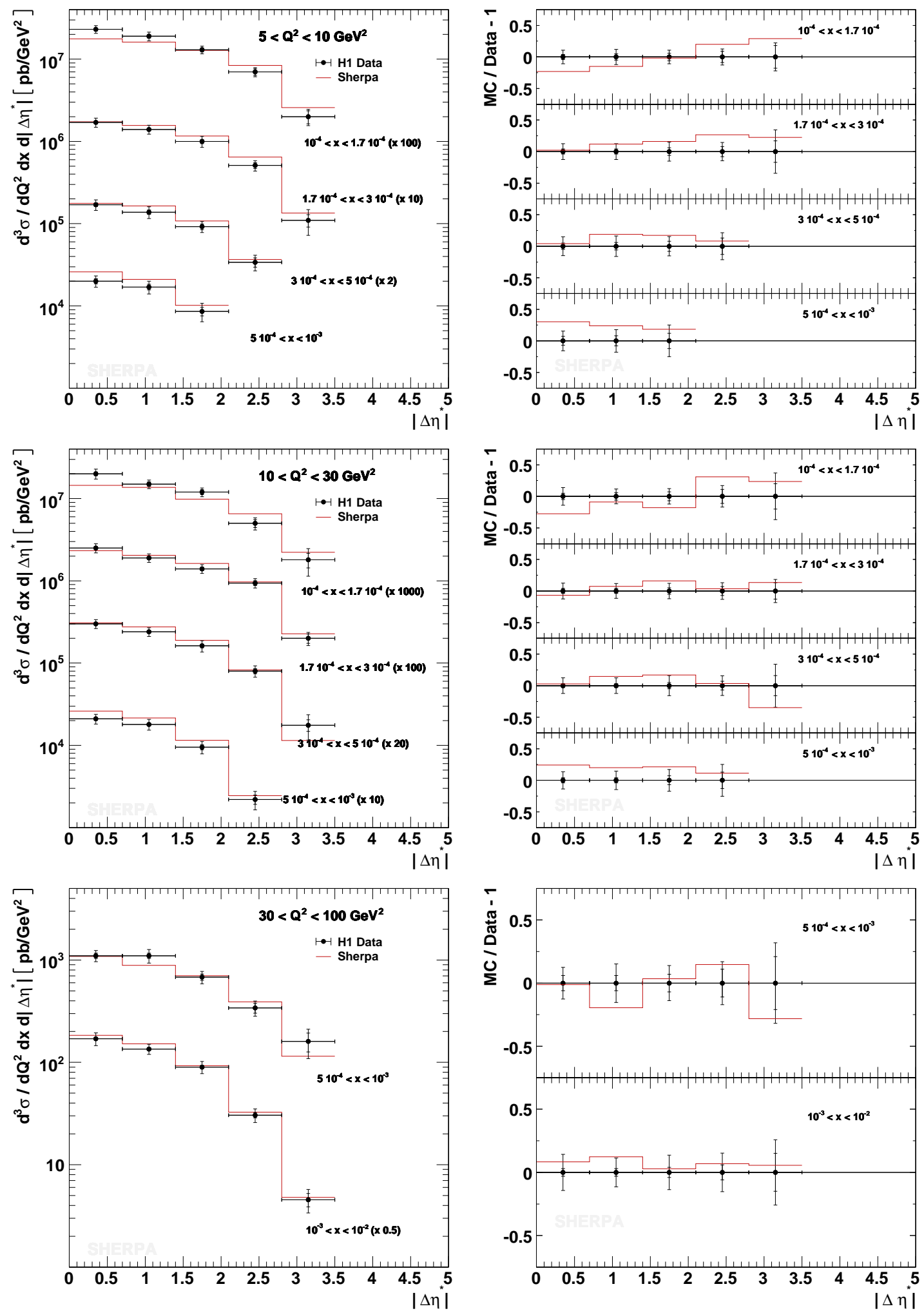

Figure 14: The di-jet differential cross section for $\Delta=2 \mathrm{GeV}$ as a function of $\left|\Delta \eta^{*}\right|$ in bins of $x$ and $Q^{2}$, measured by the H1 Collaboration [55]. 


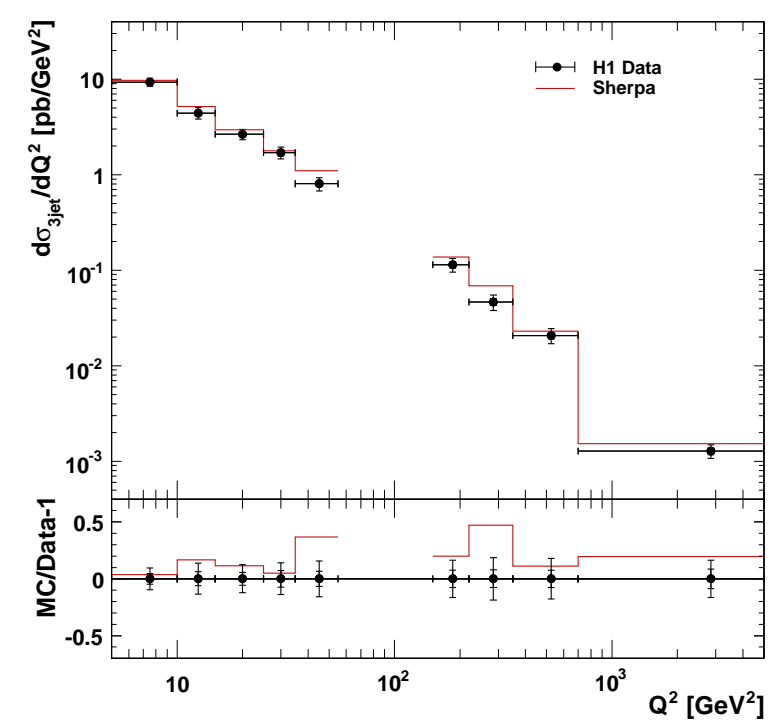

(a)

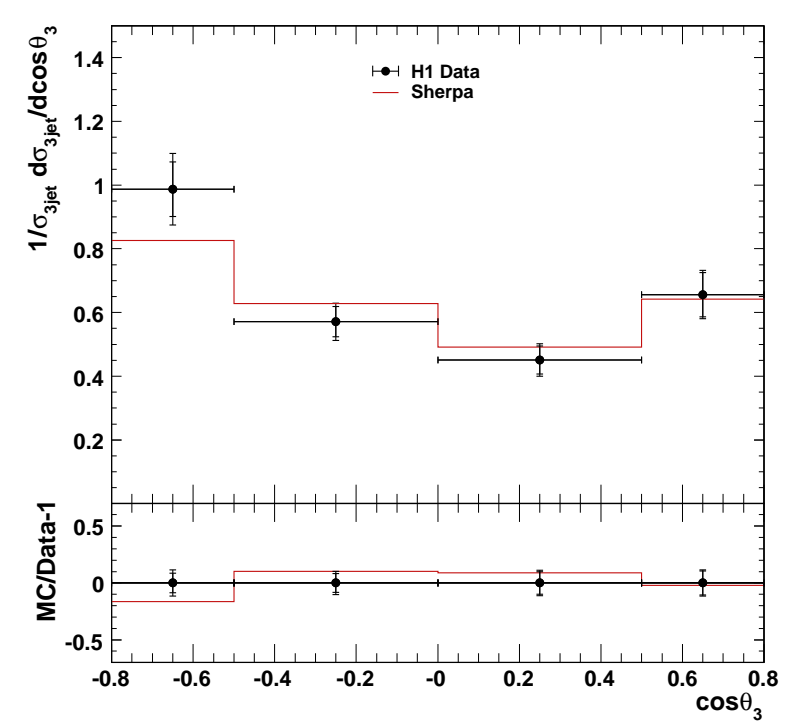

(c)

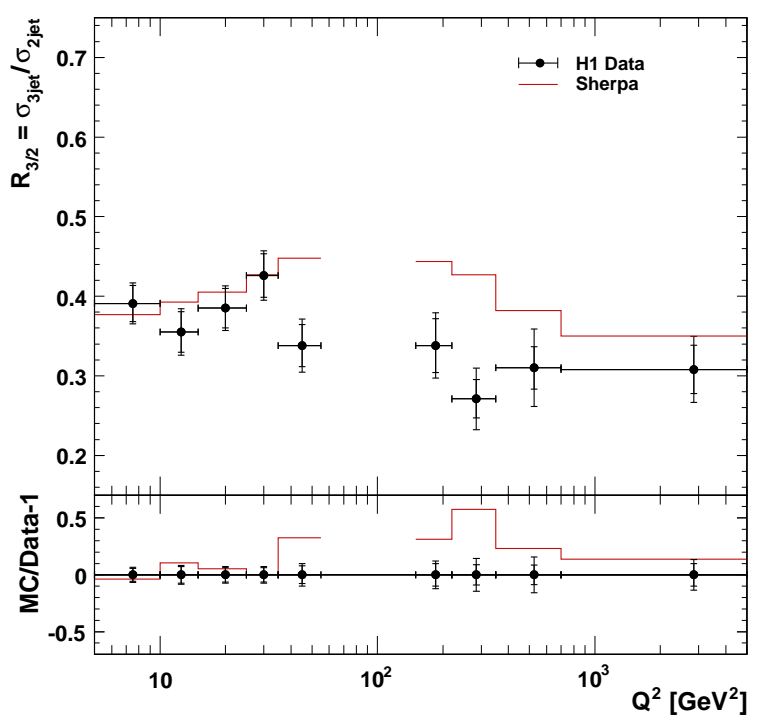

(b)

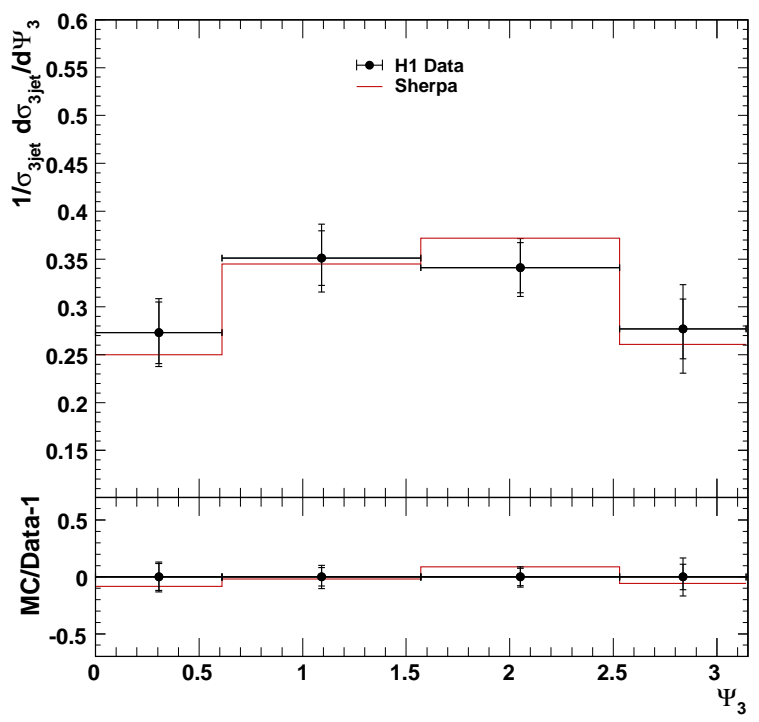

(d)

Figure 15: The three-jet cross section as a function of $Q^{2}(\mathrm{a}), \cos \theta_{3}$ (c) and $\psi_{3}(\mathrm{~d})$ and the ratio of the three- over the two-jet rate as a function of $Q^{2}$ (b), measured by the H1 Collaboration [56]. 


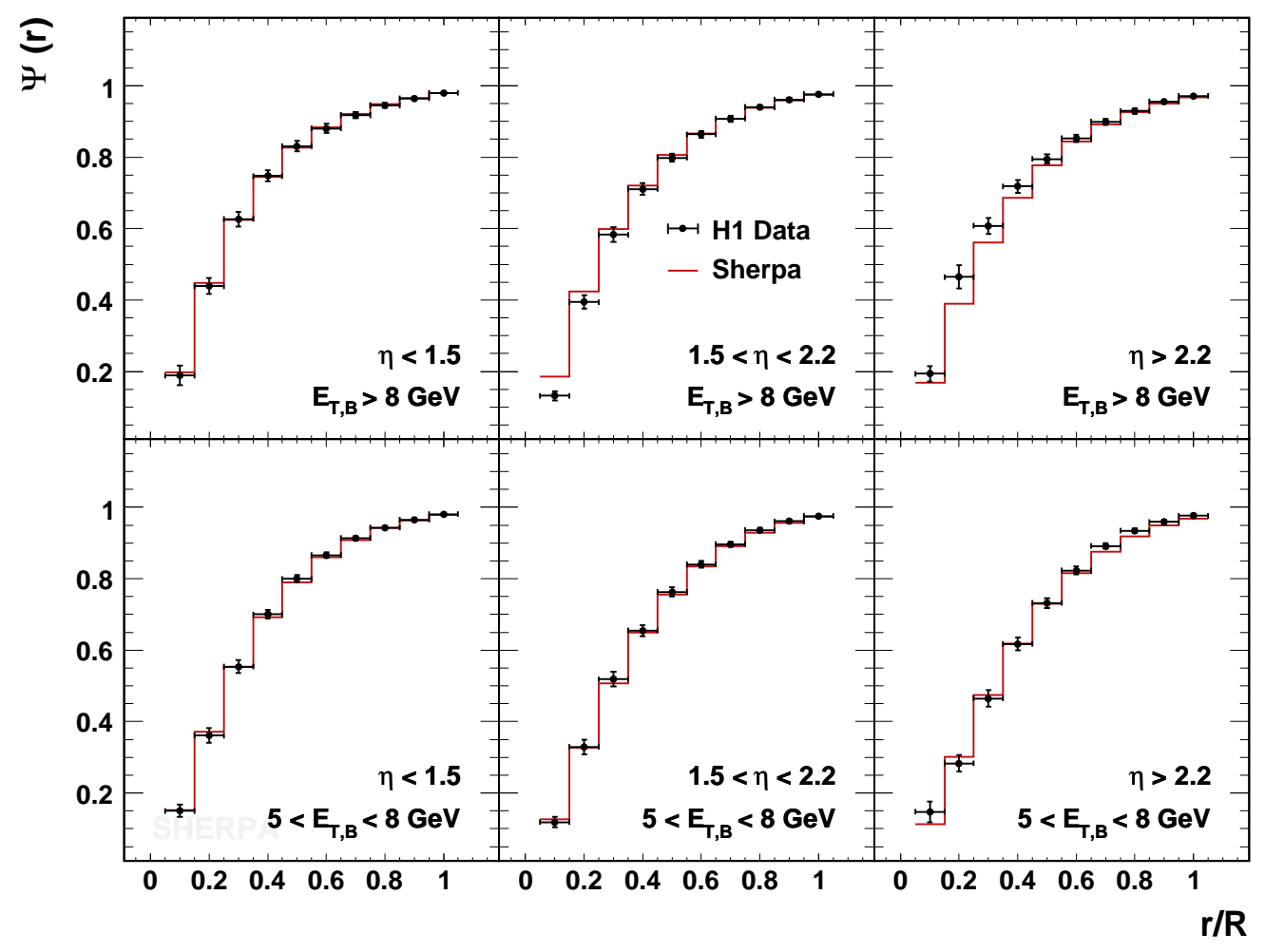

Figure 16: Jet shapes in bins of jet transverse energy and jet pseudorapidity in the Breit frame, measured by the H1 Collaboration [58].

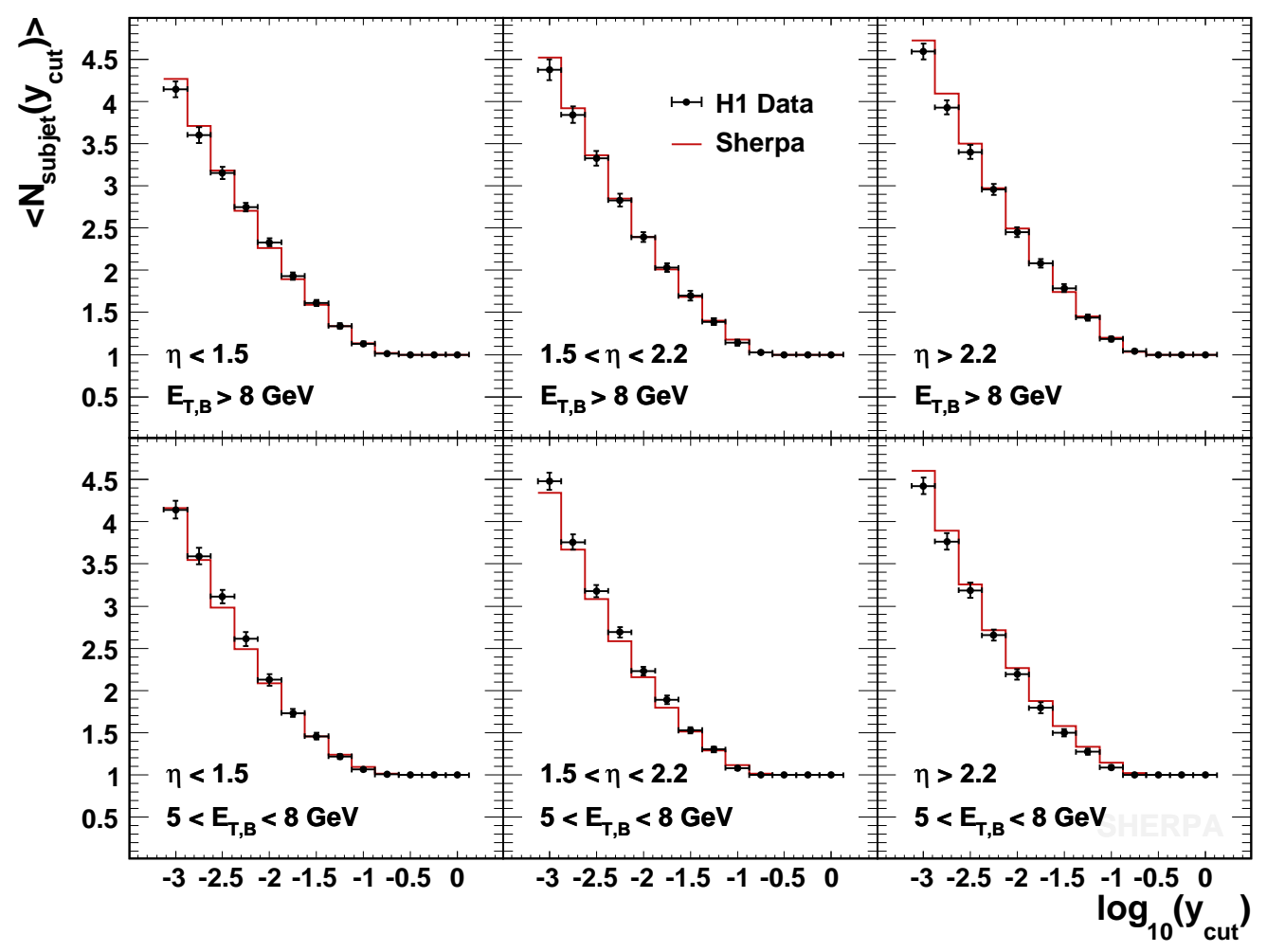

Figure 17: Sub-jet rates as a function of the resolution parameter $y_{\text {cut }}$ in bins of jet transverse energy and jet pseudorapidity in the Breit frame, measured by the H1 Collaboration [58]. 


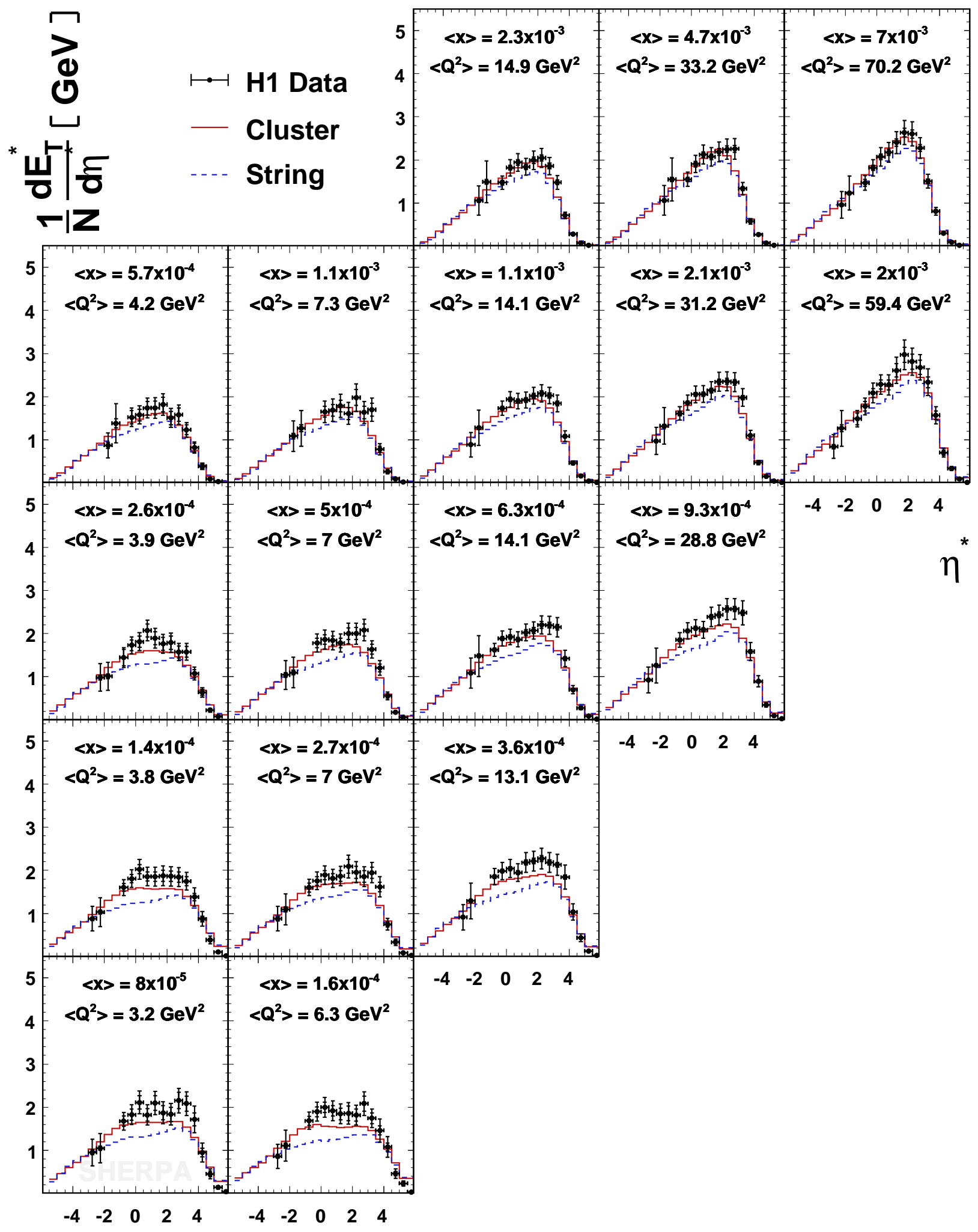

Figure 18: Transverse energy flows measured by the H1 Collaboration [61]. The histogram labeled "Cluster" displays results obtained with the cluster hadronisation model of [33], while "String" shows predictions of the Lund string hadronisation [31]. 


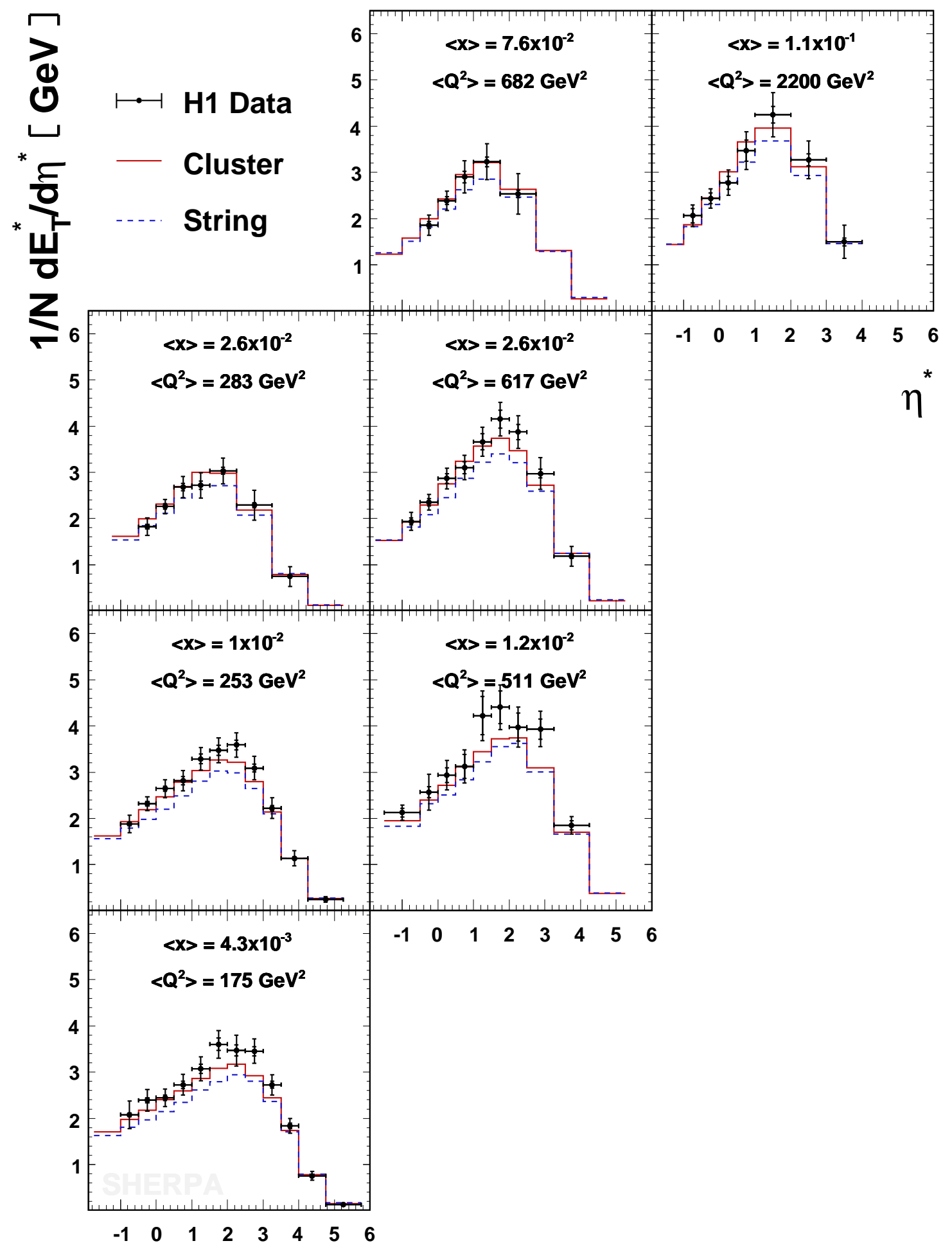

Figure 19: Transverse energy flows measured by the H1 Collaboration [61]. See Fig. 17 for notation. 


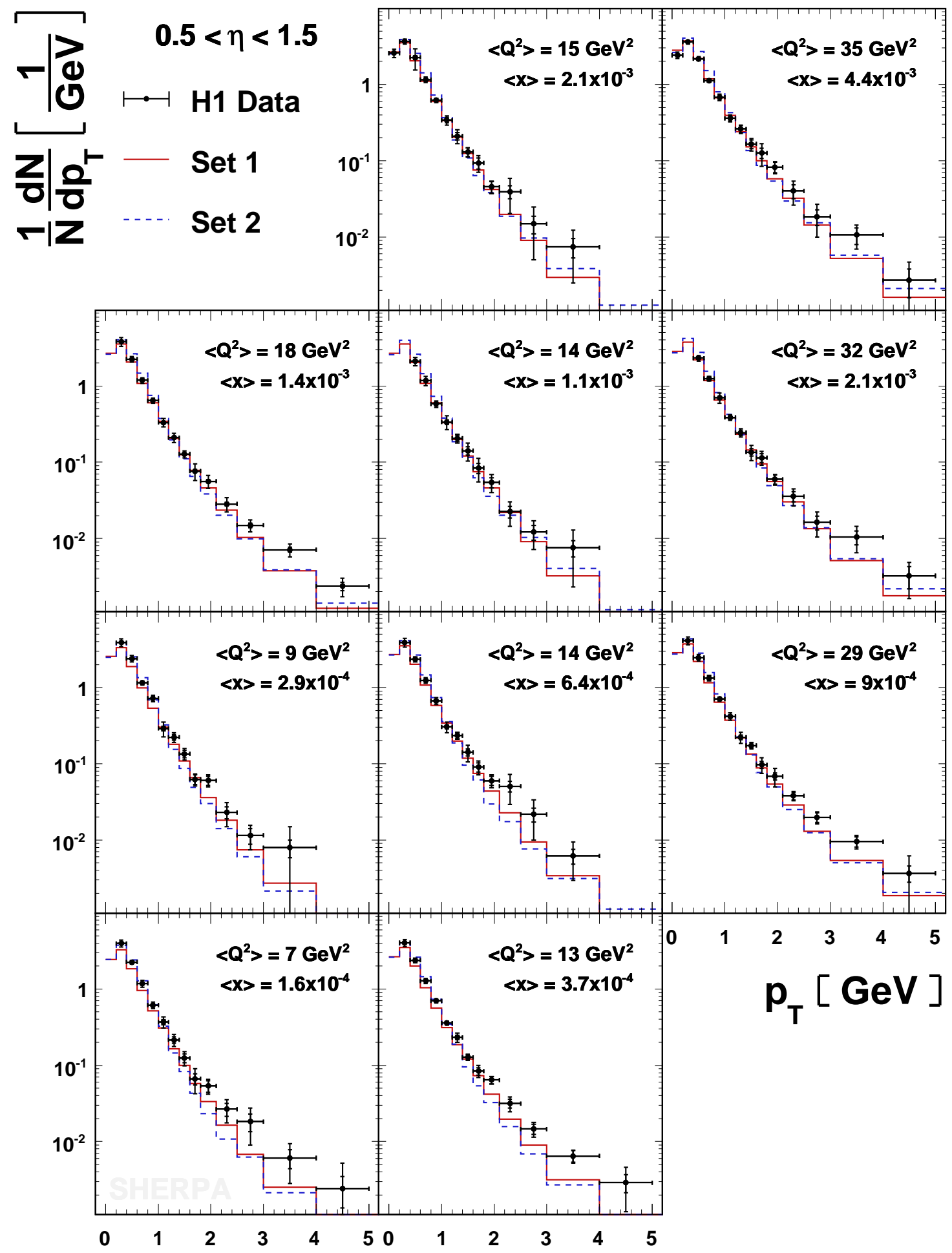

Figure 20: Charged particle transverse momentum spectra measured by the H1 Collaboration [62]. The histogram labeled "Set 1" shows predictions from the cluster hadronisation model of [33] in combination with the NNPDF 1.2 PDF set [4], while "Set 2" displays predictions from the Lund string hadronisation [31] in combination with the CTEQ 6L1 PDF set [67]. 


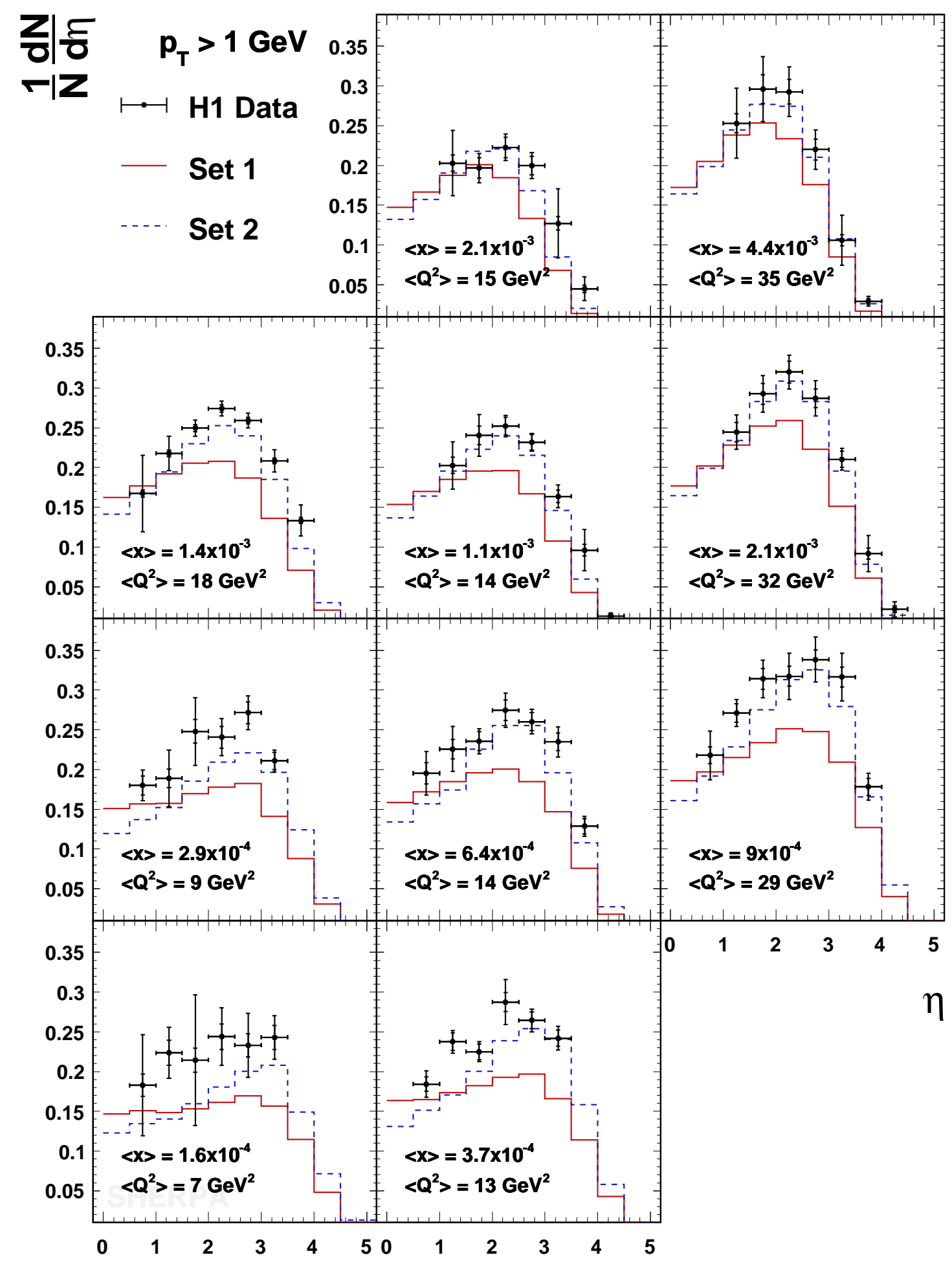

Figure 21: Charged multiplicity flow for single-particle transverse momenta larger than $1 \mathrm{GeV}$, measured by the H1 Collaboration [62]. See Fig. 19 for notation. 


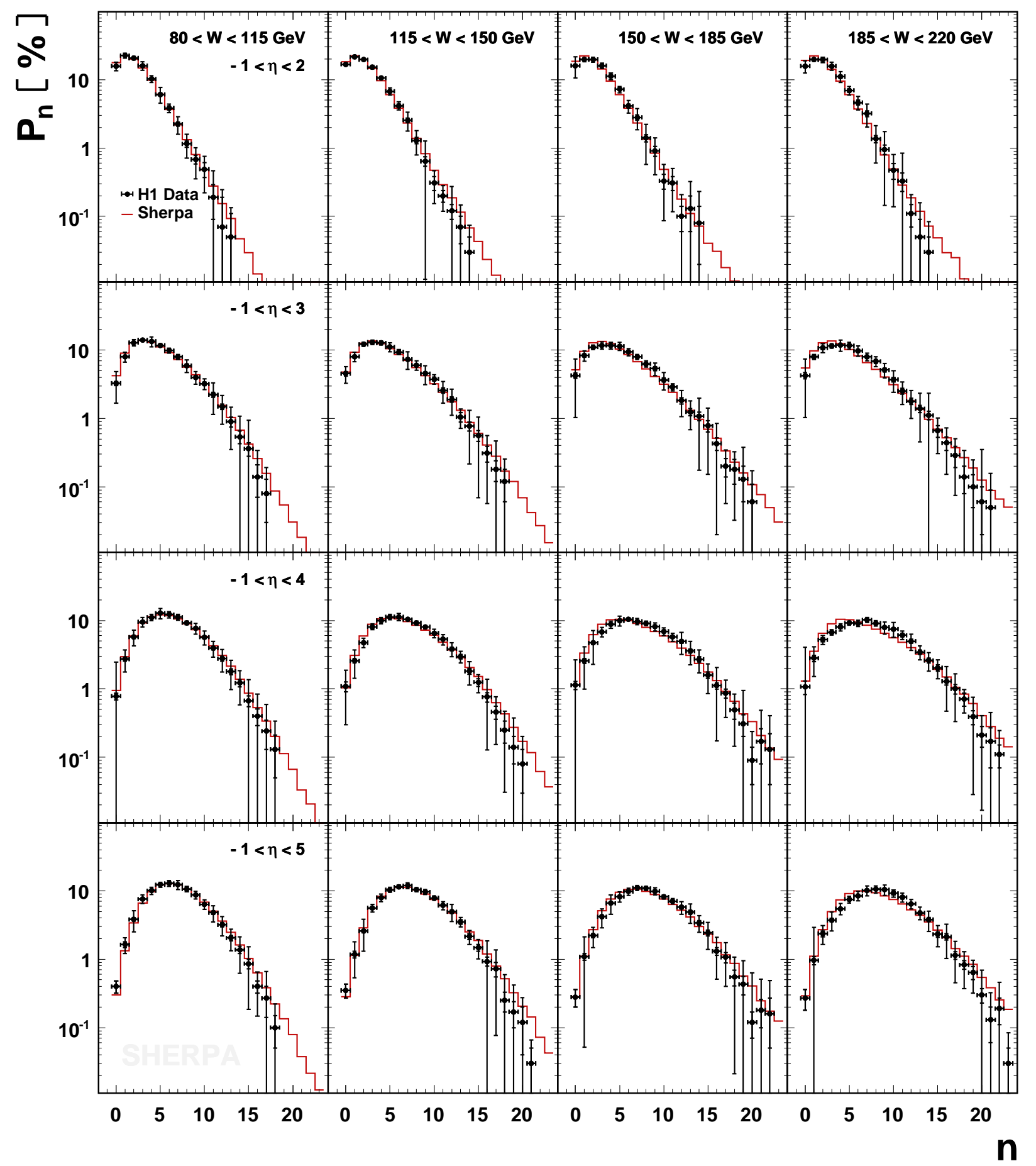

Figure 22: Charged multiplicity distributions in bins of $\eta$ and $W$, measured by the H1 Collaboration [64]. 